\title{
Strategies for the Efficient Synthesis of Biheterocyclic 5-[2-(Trifluoromethylheteroaryl)-ethyl]-1,3,4-oxadiazoles from Levulinic Acid
}

\author{
Juliana L. Malavolta, ${ }^{a}$ Leandro M. Frigo, ${ }^{b}$ Sidnei Moura, ${ }^{c}$ Darlene C. Flores ${ }^{d}$ and \\ Alex F. C. Flores $*$, \\ ${ }^{a}$ Departamento de Química, Universidade Federal de Santa Maria, 97105-900 Santa Maria-RS, Brazil \\ ${ }^{b}$ Instituto Federal Farroupilha, 97420-000 São Vicente do Sul-RS, Brazil \\ 'Instituto de Biotecnologia, Universidade de Caxias do Sul, 95070-560 Caxias do Sul-RS, Brazil \\ ${ }^{d}$ Escola de Química e Alimentos, Universidade Federal de Rio Grande, 96203-900 Rio Grande-RS, Brazil
}

\begin{abstract}
The synthesis of 5-[2-(trifluoromethylheteroaryl)-ethyl]-1,3,4-oxadiazoles derived from levulinic acid is reported. Cyclocondensations [4 +1] between four different 5-[2-(trifluoromethylheteroaryl) propionylhydrazides derived from methyl 7,7,7-trifluoro-4-methoxy-6-oxo-4-heptenoate obtained from levulinic acid, and electrophilic orthoesters $\mathrm{RC}\left(\mathrm{OR}^{1}\right)_{3}$ (where $\mathrm{R}=\mathrm{H}, \mathrm{Me}, \mathrm{Ph}$ ) and $\mathrm{CS}_{2}$ were carried out in a mild medium. Good yields (69-96\%) of isolated products were obtained. The structures of the new ethylene-spaced biheterocycles were characterized using ${ }^{1} \mathrm{H}$ and ${ }^{13} \mathrm{C}$ nuclear magnetic resonance (NMR) spectroscopy and electrospray ionization coupled to tandem mass spectrometric (ESI MS/MS) data.
\end{abstract}

Keywords: heterocycles, oxadiazoles, pyrimidines, pyrazoles, levulinic acid

\section{Introduction}

The progressive depletion of fossil resources, and fluctuations in their prices, are promoting a shift from fossil to renewable materials as feedstock for the production of energy, fuels, and chemicals. Nowadays, only $5 \%$ of all chemicals produced worldwide are derived from renewable resources. However, cellulosic biomass, hitherto underutilized, can be converted into value-added chemicals by acid hydrothermal treatment. ${ }^{1,2}$ Levulinic acid (LA) is a major product of controlled degradation of hexose sugars by acids. The LA molecule contains highly reactive carbonyl and carboxyl groups that can be transformed in a variety of ways, serving as a versatile building block for the synthesis of value-added organic compounds. ${ }^{3}$

On the other hand, the synthesis of $\mathrm{CF}_{3}$-containing heterocycles has received intensive attention because of the important functions these compounds play in agrochemicals, pharmaceuticals, and specialized materials. It is well known that adding a fluorinated group to certain compounds will modify their physicochemical profiles,

\footnotetext{
*e-mail: alexflores@ furg.br
}

increasing their lipophilicity and metabolic stability., Among heterocyclic compounds, 1,3,4-oxadiazole has become an important construction template for the development of new drugs. It has attracted interest in medicinal chemistry as a bioisostere for carboxylic acids, esters, and carboxamides. ${ }^{4}$ The ability of 1,3,4-oxadiazole heterocyclic compounds to undergo various chemical reactions has made them important for molecule planning, which has enormous biological potential. ${ }^{6-13}$

Recently we have reported the synthesis of methyl 1,1,1-trifluoro-4-methoxy-6-oxo-4-heptenoate as a building block for trifluoromethyl containing heterocyclic systems. This substrate has three electrophilic centers allowing its use in $[4+1],[3+2]$, and [3+3] cyclocondensation processes, as shown in Scheme 1. ${ }^{14-18}$

Our ongoing interest in producing and understanding the biological activities of halogenated heterocyclic systems led us to study strategies for synthesizing a diversity of biheterocyclic systems, such as 5-[2-(trifluoromethylheteroaryl)-ethyl]-1,3,4-oxadiazoles (4-7), with an ethylene spacer between heterocyclic nuclei, from methyl 3-(trifluoromethylheteroaryl)propanoates, where trifluoromethylheteroaryl is 5(3)-trifluoromethyl-1H-pyrazol3(5)-yl, 2-phenyl-6-trifluoromethyl pyrimidin-4-yl, 




Scheme 1. Cyclocondensations from methyl 7,7,7-trifluoro-4-methoxy-6-oxo-4-heptenoate (1).

2-thiomethyl-6-trifluoromethylpyrimidin-4-yl, or 2-methyl7-trifluoromethyl pyrazolo[1,5-a]pyrimidin-5-yl.

\section{Results and Discussion}

The starting methyl 7,7,7-trifluoro-4-methoxy-6-oxo4-heptenoate (1) was synthesized by acylation of methyl 4,4-dimethoxypentanoate with trifluoroacetic anhydride in pyridine and dichloromethane, which resulted in good yields of $90 \%$. No detectable amounts of other acylation products were observed. The in situ formation of kinetic enol ether under acylation conditions was discussed in a previous study ${ }^{19}$ Following the conventional route to $\mathrm{CF}_{3}$-containing $1 \mathrm{H}$-pyrazole, the reaction of hydrazine hydrochloride with $\mathbf{1}$ in ethanol proceeded to give nearly quantitative yields of methyl 3-(5-trifluoromethyl-1 $H$-pyrazol-3-yl)propanoate (2a). ${ }^{16}$ For cyclocondensations between precursor $\mathbf{1}$ and amidine salts (hydrochloride or sulfate), we started the process based on a previous report on cyclocondensation [3+3] of 1,1,1-trifluoro-4-alkoxy-3-alken-2-ones and amidines under basic $\mathrm{NaOH}$ or alkoxy (methoxy, ethoxy) catalysis. ${ }^{18}$ Initially, the cyclocondensation between 1 and 2-methyl-2-thiopseudourea sulfate was carried out in methanol via catalysis with $1 \mathrm{M} \mathrm{NaOH}$ aqueous solution at $25^{\circ} \mathrm{C}$ for $1 \mathrm{~h}$. This led to a good yield of $66 \%$ for pure methyl 3-(2-thiomethyl-6-trifluoromethylpyrimidin-4-yl) propanoate $(\mathbf{2 c})$. These conditions were extended to cyclocondensations between the precursor $\mathbf{1}$ and benzamidine hydrochloride, leading to methyl 3-(2-phenyl6-trifluoromethyl pyrimidin-4-yl)propanoate (2b) at a good yield of $67 \% .{ }^{20}$ The reaction between $\mathbf{1}$ and 3-amino-5-methyl-1H-pyrazole was performed under conditions described in a previous report, ${ }^{21}$ and there was exclusive formation of 2-methyl-5-(methylpropanoate-3-yl)7-trifluoromethylpyrazolo[1,5-a]pyrimidine (2d) at $89 \%$ yield. The synthetic strategy adopted to obtain the target products involves the conversion of methyl 3-heteroarylpropanoates $\mathbf{2 a - d}$ to key intermediate hydrazides 3a-d by refluxing with hydrazine hydrate in ethanol, a well-described procedure for diverse ester substrates. ${ }^{22}$

These hydrazides were reacted with trialkylorthoesters (orthoformate, orthoacetate, and orthobenzoate) to obtain 5-[2-(trifluoromethylheteroaryl)-ethyl]1,3,4-oxadiazoles (4-6) under mild conditions without other solvent (Table 1, Scheme 2). ${ }^{23}$ Initially, the cyclocondensation between $\mathbf{3 c}$ and triethyl orthoacetate was carried out in ethanol reflux at a 1:1 stoichiometric ratio for $24 \mathrm{~h}$, leading to a mixture of product $\mathbf{5 c}$ and reagents. Increasing the proportion of orthoacetate and performing the reaction at a stoichiometric ratio of 1:3 (3c:orthoacetate) 
in refluxing ethanol made it possible to isolate product $\mathbf{5 c}$ at a reasonable yield of $52 \%$. However, the best condition was using an excessive amount of orthoacetate (6 to 10 mole equivalents) without another solvent, at $80^{\circ} \mathrm{C}$, which led to a $92 \%$ yield of isolated $\mathbf{5 c}$ (Table 1, entries 1-3). These conditions were extended to cyclocondensations between all precursors 3 and orthoesters, leading to 5-(2-(trifluoromethylheteroaryl)ethyl)-1,3,4-oxadiazoles 4-6 at very good yields. The reactions proceeded smoothly and cleanly under mild conditions and no side reactions were observed in any series.

The preparation of the series of 5-(2-(trifluoromethylheteroaryl)ethyl)-1,3,4-oxadiazole-2-thiol 7b-d was carried out using a simple one-pot procedure that involves reacting the respective hydrazide 3 with $\mathrm{CS}_{2}$ under strong basic conditions followed by acidification with $\mathrm{HCl}$ solution, as already described in the literature. ${ }^{24,25}$ Using an adaptation of the experimental methodology described by El-Din Mohamed et al. ${ }^{7}$ led to products $7 \mathbf{a}-\mathbf{d}$ at excellent yields.

The functionalization of 1,3,4-oxadiazole-2-thiols by alkylation and acylation reactions of the -SH group is not as common in the literature as one would expect. Hence, for this reason, and also because such a procedure may result in compounds with a broad potential spectrum of pharmacological activities, we decided to conduct such studies. To this end, we carried out alkylation reactions with 2-bromoacetophenone and acylation reactions with acetyl anhydride and trichloroacetyl chloride of the series 7b-d, as summarized in Scheme 3, using adaptations of experimental methodologies described in the literature. ${ }^{26,27}$

The characterization data of all of the synthesized compounds are given in the Experimental section. All of the newly synthesized compounds gave satisfactory analyses for the proposed structures, which were confirmed based on their 1D / 2D NMR (nuclear magnetic resonance) and HRMS (high resolution mass spectrometry) spectral data. For all series of biheterocyclic products, a multiplet due to an ethylene chain spacer between 3-4 ppm was observed in the ${ }^{1} \mathrm{H}$ NMR spectra, and the shape varied from a singletlike signal to two well-defined triplets (see Supplementary Information). In the ${ }^{13} \mathrm{C}$ NMR spectra are characteristic for all series of the product isolated the signals from ethylene spacer at $\delta 22$ and $32 \mathrm{ppm}$ and at compatible chemical shifts, the signals as quartets from the carbons coupling to the fluorine atoms.

For example, the signal related to methylenes from the ethylene spacer of the 2-[2-(5-trifluoromethyl$1 H$-pyrazol-3-yl)ethyl]-1,3,4-oxadiazole (4a) and 2-methyl-5-[2-(2-phenyl-6-trifluoromethylpyrimidin-4-yl) ethyl]-1,3,4-oxadiazole (5b) consisted of enlarged singlets at $\delta 3.26$ and $3.44 \mathrm{ppm}$, respectively. ${ }^{16}$ The signal for $\mathrm{H} 4$ from the aromatic $1 H$-pyrazol ring of product $\mathbf{4 a}$ was at

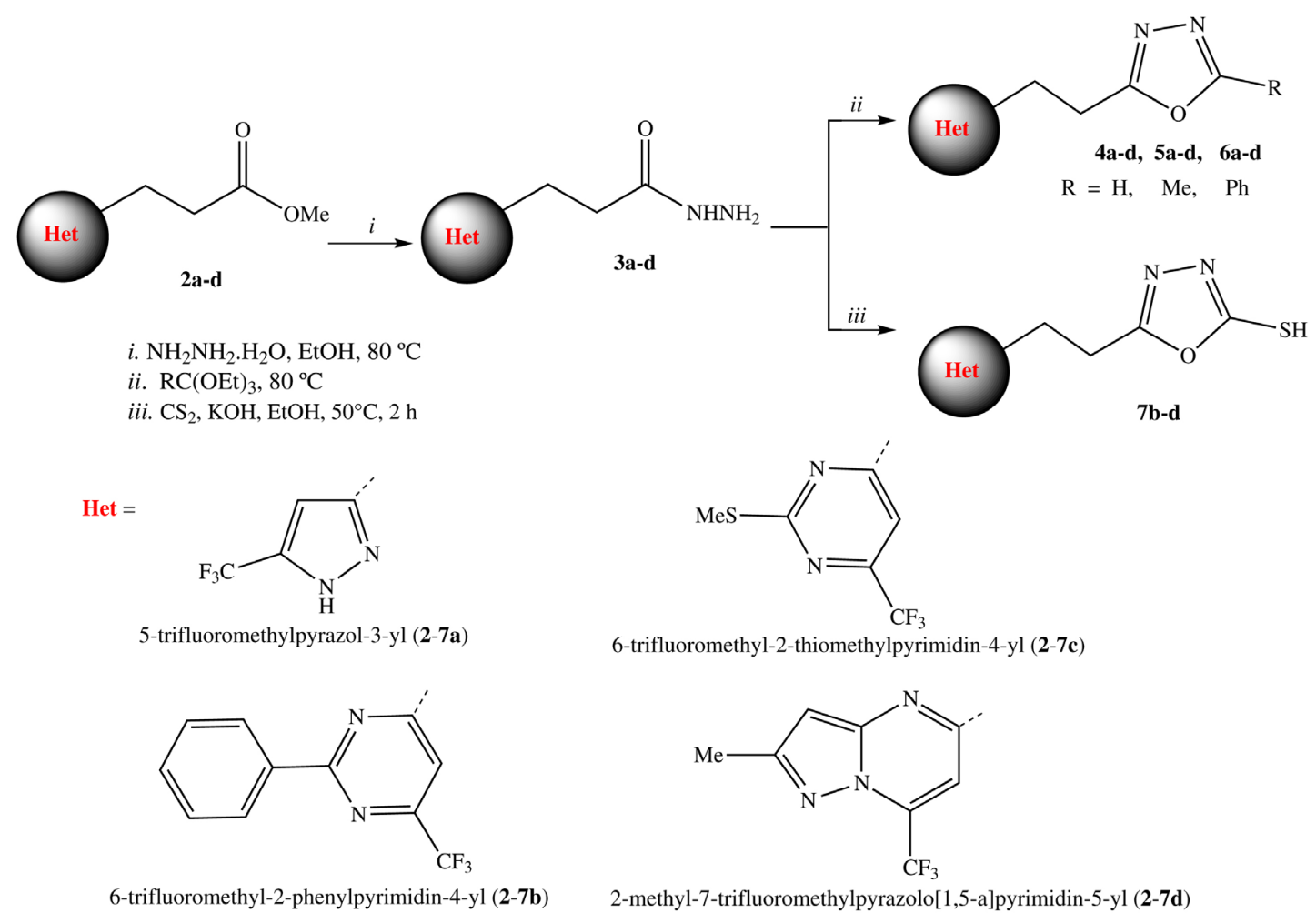

Scheme 2. Route to 5-[2-(trifluoromethylheteroaryl)-ethyl]-1,3,4-oxadiazoles 4, 5, 6, 7. 
Table 1. Reactional condition optimization to [4+1] cyclocondensation between $\mathbf{3 c}$ and triethyl orthoacetate

\begin{tabular}{lccccc}
\hline entry & Solvent & $\begin{array}{c}\text { Ratio } \\
\text { 3c:orthoacetate }\end{array}$ & Temperature $/{ }^{\circ} \mathrm{C}$ & time $/ \mathrm{h}$ & Yield $/ \%$ \\
\hline 1 & ethanol & $1: 1$ & 78 & 24 & $-\mathrm{a}$ \\
2 & ethanol & $1: 3$ & 78 & 24 & 52 \\
3 & orthoacetate & $1: 10$ & 100 & 16 & 95 \\
4 & orthoacetate & $1: 6$ & 80 & 12 & 92 \\
5 & orthoacetate & $1: 3$ & 80 & 16 & 92 \\
\hline
\end{tabular}

${ }^{\mathrm{a}}$ Mixture of $\mathbf{5 c}$ and starting reagents, 3:7.

$\delta 6.37 \mathrm{ppm}$, while that for $\mathrm{H} 5$ of the 2-phenylpyrimidine ring of product $\mathbf{5 b}$ had a signal at $\delta 7.41 \mathrm{ppm}$. The ${ }^{13} \mathrm{C}$ NMR spectra showed the characteristic signals for each derivative series. The quartets related to the $\mathrm{CF}_{3}$ group attached to heteroaromatic rings were observed at about $\delta 120 \mathrm{ppm}$ with ${ }^{3} J_{\mathrm{CF}} 275-276 \mathrm{~Hz}$, the quartet signal related to $\mathrm{C}_{-} \mathrm{CF}_{3}$ from the aromatic $1 H$-pyrazole ring was at about $\delta 142 \mathrm{ppm}$ with $J_{\mathrm{CF}} 38 \mathrm{~Hz}$, and that related to $\mathrm{C} 5$ from the pyrimidine ring was at about $\delta 113 \mathrm{ppm}$ with ${ }^{3} J_{\mathrm{CF}} 34-36 \mathrm{~Hz}$. The signals related to ethylene methylenes appeared at 21 and $34 \mathrm{ppm}$, and signals from two aromatic carbonyl-like carbons from 1,3,4-oxadiazole appeared in the characteristic deshielded region of 150-175 ppm (see Supplementary Information).

We also tested other approaches reported in the literature for obtaining a diversity of 5-[2-(trifluoromethylheteroaryl)ethyl]-1,3,4-oxadiazoles, for example, from the oxidation

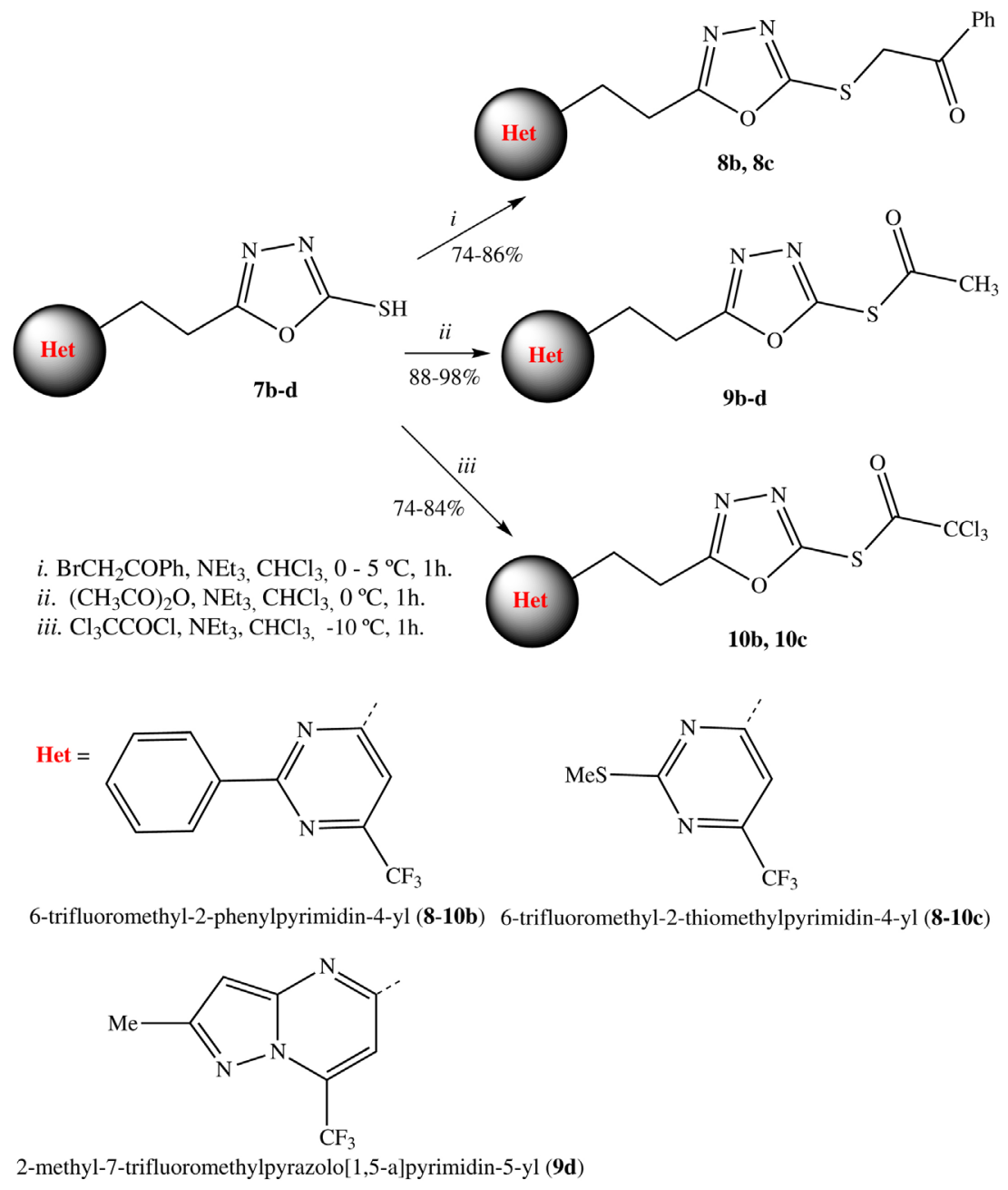

Scheme 3. S-Alkylation/acylation reactions of the 5-(2-(trifluoromethylheteroaryl)ethyl)-1,3,4-oxadiazole-2-thiol derivatives. 
Table 2. Other methodologies tested for 5(2)-[2-(trifluoromethylheteroaryl)ethyl]-1,3,4-oxadiazole synthesis from levulinic acid hydrazide derivatives

\begin{tabular}{lccccc}
\hline entry & Precursor & Methodology & Product & Yield $/ \%$ & Reference \\
\hline 1 & $\mathbf{1 1 b}$ & $N$-acylhydrazone oxidation with chloramine-T & $\mathbf{1 1 b}$ & - & 28 \\
2 & $\mathbf{1 1 b}$ & $N$-acylhydrazone oxidation with TCCA & $\mathbf{1 2 b}$ & 79 & 29 \\
3 & $\mathbf{1 3 b}$ & $\mathrm{SOCl}_{2} N$-acetylhydrazide dehydration & $\mathbf{1 3 b}$ & - & 30 \\
\hline
\end{tabular}

of $N$-acylhydrazones derived from hydrazides $\mathbf{3 b}$-d and $p$-chlorobenzaldehyde (11b-d) with trichloroisocyanuric acid (TCCA) or chloramine- $\mathrm{T}$, and from diacylhydrazides derived from $\mathbf{3 b}-\mathbf{d}$ and acetic anhydride $(\mathbf{1 2 b}, \mathbf{c})$ with thionyl chloride (Table 2). However, the target products, 5-(2-(trifluoromethylheteroaryl)ethyl)-1,3,4-oxadiazole, were not formed. One procedure, reported earlier by Pore et al. ${ }^{29}$ was successfully reproduced by reacting $\mathrm{N}$-acylhydrazones 11b-d with TCCA in EtOH, however, it did not lead to the products of oxidative cyclization, the 1,3,4-oxadiazoles derivatives, but only to ethyl 3-(trifluoromethylheteroaryl)propanoates 12b-d as well (Scheme 4). The reaction condition optimization was conducted between $N$-acylhydrazone 11b and TCCA in $\mathrm{EtOH}$ at $25^{\circ} \mathrm{C}$ (Table 3), under these conditions TCCA just catalyzes the ethanolysis of the hydrazide bond.

Table 3. Optimization of the reaction conditions between $N$-acylhydrazone $\mathbf{1 1 b}$ and TCCA (trichloroisocyanuric acid) in $\mathrm{EtOH}$ at $25^{\circ} \mathrm{C}$

\begin{tabular}{lccc}
\hline entry & $\begin{array}{c}\text { 11b:TCCA } \\
\text { ratio }\end{array}$ & time / min & Yield $^{\mathrm{a} / \%}$ \\
\hline 1 & $1: 1$ & 60 & 79 \\
2 & $1: 1$ & 30 & 76 \\
3 & $1: 1$ & 10 & 76 \\
4 & $1: 0.5$ & 10 & 71 \\
5 & $1: 0.25$ & 10 & 64 \\
6 & $1: 0.25$ & 20 & 64 \\
7 & $1: 0.25$ & 60 & 70 \\
\hline
\end{tabular}

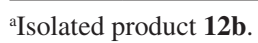

\section{Conclusions}

In conclusion, the dielectrophilic precursor methyl 7,7,7-trifluoro-4-methoxy-6-oxo-4-heptenoate is versatile and efficient in $[3+3]$ and $[3+2]$ cyclocondensations with different dinucleophiles, leading to a variety of 3-(trifluoromethylheteroaryl)-propanoates (2a-d) at a scale of grams, which can be converted into respective 3-(trifluoromethylheteroaryl) propanoylhydrazides (3a-d). These, in turn, are new dinucleophilic substrates that can be reacted with different monoeletrophilic blocks to obtain new ethylene-spaced biheterocyclic systems.
Here, we specifically described the synthesis of (trifluoromethylheteroaryl)propanoyl hydrazides (3a-d) and their cyclocondensation with trialkyl orthoformates and carbon disulfide as an efficient protocol for the preparation of diverse, novel 5(2)-[2-(trifluoromethylheteroaryl)-ethyl]1,3,4-oxadiazoles (4-10) at good yields. These compounds are interesting structural analogs to central nervous system chemical mediators, making them good subjects for the study of biological activity. Furthermore, heterocyclic imine nitrogens may be interesting modulators of the electronic characteristics of metals in compounds used in luminescent devices.

\section{Experimental}

${ }^{1} \mathrm{H},{ }^{13} \mathrm{C},{ }^{19} \mathrm{FNMR}$ spectra were collected at $300 \mathrm{~K}$ using a Bruker $5 \mathrm{~mm}$ dual probe on a Bruker DPX 400 spectrometer $\left({ }^{1} \mathrm{H}\right.$ at $400.13 \mathrm{MHz},{ }^{19} \mathrm{~F}$ at $376.4 \mathrm{MHz},{ }^{13} \mathrm{C}$ at $\left.100.62 \mathrm{MHz}\right)$. Chemical shifts $(\delta)$ are given in parts per million (ppm) from tetramethylsilane (TMS), and coupling constants $(J)$ are given in Hz. Melting points were determined using open capillaries on an Electrothermal Mel-Temp 3.0 apparatus and are uncorrected. Electrospray ionization (ESI) high-resolution mass spectra were determined using an Agilent 6460 Triple Quadrupole connected to a 1200 series LC and equipped with a solvent degasser, binary pump, column oven, auto-sampler, and an ESI source. The Agilent QQQ 6460 tandem mass spectrometer (MS/MS) was operated in the positive jet stream ESI mode. Nitrogen was used as a nebulizer, turbo (heater) gas, curtain gas, and collision-activated dissociation gas. The capillary voltage was set to $+3500 \mathrm{~V}$ and the nozzle voltage was set to $+500 \mathrm{~V}$. The ion source gas temperature was $300{ }^{\circ} \mathrm{C}$ with a flow rate of $5 \mathrm{~L} \mathrm{~min}^{-1}$. The jet stream sheath gas temperature was $250{ }^{\circ} \mathrm{C}$ with a flow rate of $11 \mathrm{~L} \mathrm{~min}^{-1}$. All samples were infused into the ESI source at a $5 \mu \mathrm{L} \mathrm{min}{ }^{-1}$ flow rate. Data were acquired in positive MS total ion scan mode (mass scan range $\mathrm{m} / \mathrm{z}, 50-650$ ) and in positive MS/MS product ion scan mode. The mass spectra recorded were evaluated using the Qualitative Analysis software from Agilent Technologies. CHN elemental analyses were performed on a PerkinElmer $2400 \mathrm{CHN}$ elemental analyzer (São Paulo University (USP), Brazil). 

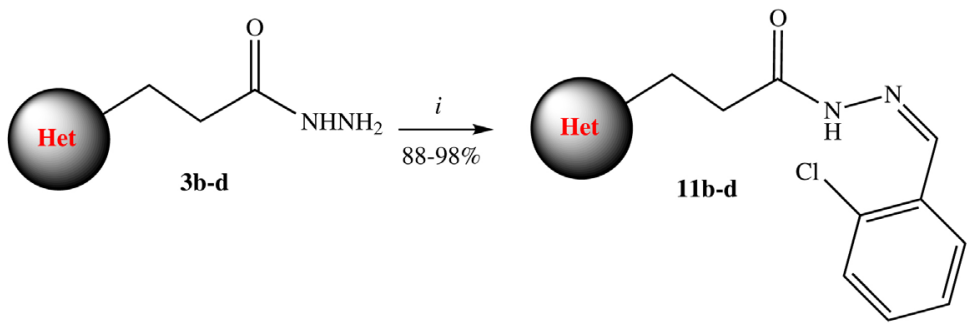

i. $2-\mathrm{ClC}_{6} \mathrm{H}_{4} \mathrm{CHO}, \mathrm{EtOH}, 50^{\circ} \mathrm{C}, 6 \mathrm{~h}$. ii. TCCA, EtOH, $\mathrm{CHCl}_{3}, 25^{\circ} \mathrm{C}, 30^{\prime}$.
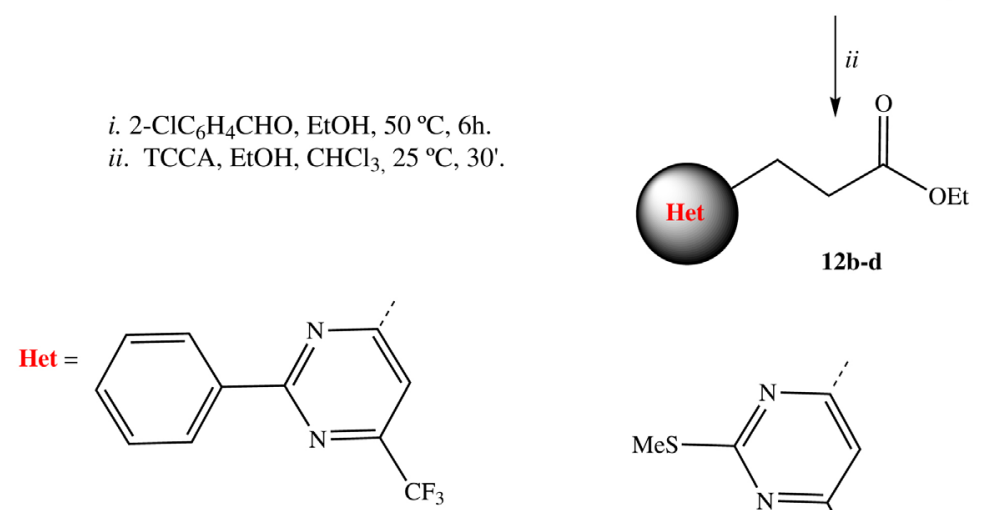

6-trifluoromethyl-2-phenylpyrimidin-4-yl $(\mathbf{3}, \mathbf{1 1}, \mathbf{1 2 b})$
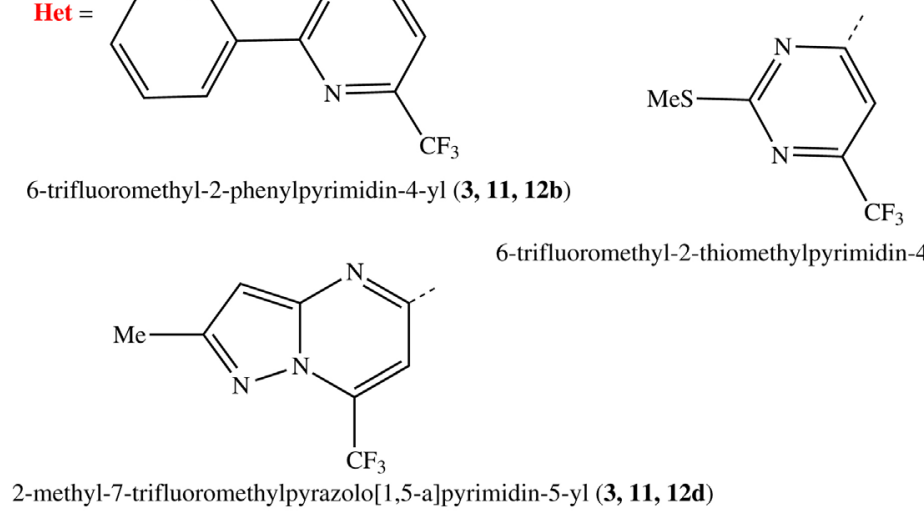

Scheme 4. Synthesis and reactions of $N$-acylhydrazones and TCCA in EtOH.

General procedure for the synthesis of 5-substituted1,3,4-oxadiazoles (4a-d, 5a-d, 6a-d)

A solution of the prepared hydrazide $3 \mathbf{a}-\mathbf{d}(5 \mathrm{mmol})$ in the respective orthoester (30-50 mmol) was kept under stirring at $80{ }^{\circ} \mathrm{C}$ until the starting hydrazide was fully consumed, monitored by thin layer chromatograph (TLC), $16 \mathrm{~h}$. After cooling, the excess orthoester was evaporated under reduced pressure. The crude product 4a-d, 5a-d and 6a-d was crystallized (hexane) or chromatographed (hexane-AcOEt mixtures) to give the following:

5-[2-(5(3) Trifluoromethylpyrazol-3(5)-yl)-ethyl]1,3,4-oxadiazole (4a)

Obtained $(63 \%)$ as a colorless dense oil; ${ }^{1} \mathrm{H}$ NMR (400.13 MHz, $\left.\mathrm{CDCl}_{3}\right) \delta 3.26\left(\mathrm{~m}, 4 \mathrm{H}, \mathrm{CH}_{2}\right), 6.37$ (s, 1H), $8.41(\mathrm{~s}, 1 \mathrm{H}) ;{ }^{13} \mathrm{C} \mathrm{NMR}\left(100.62 \mathrm{MHz}, \mathrm{CDCl}_{3}\right) \delta 21.7,24.9$, $102.9,121.2\left(\mathrm{q}, J_{\mathrm{CF}} 275 \mathrm{~Hz}\right), 142.7\left(\mathrm{q}, J_{\mathrm{CF}} 36 \mathrm{~Hz}\right), 143.4$, 153.3, 165.8; $\left.{ }^{19} \mathrm{~F} \mathrm{NMR} \mathrm{(376.4} \mathrm{MHz,} \mathrm{CDCl}_{3}\right) \delta-61.7$; HRMS (FTMS (Fourier transform mass spectrometry) + pESI) $\mathrm{m} / \mathrm{z}$, calcd. for $\mathrm{C}_{8} \mathrm{H}_{7} \mathrm{~F}_{3} \mathrm{~N}_{4} \mathrm{O}\left[\mathrm{MH}^{+}\right]$: 233.0650, found: 233.0534 $\left[\mathrm{MH}^{+}\right]$. Anal. calcd. for $\mathrm{C}_{8} \mathrm{H}_{7} \mathrm{~F}_{3} \mathrm{~N}_{4} \mathrm{O} 232.16 \mathrm{~g} \mathrm{~mol}^{-1}: \mathrm{C}$, 41.39; H, 3.04; N, 24.13; found: C, 41.5; H, 3.05; N, 23.90.
2-[2-(2-Phenyl-6-trifluoromethylpyrimidin-4-yl)ethyl]1,3,4-oxadiazole (4b)

Obtained $(65 \%)$ as a yellowish solid, mp $105-107^{\circ} \mathrm{C}$; ${ }^{1} \mathrm{H}$ NMR $\left(400.13 \mathrm{MHz}, \mathrm{CDCl}_{3}\right) \delta 3.46\left(\mathrm{~m}, 2 \mathrm{H}, \mathrm{CH}_{2}\right), 3.53$ (m, 2H, $\left.\mathrm{CH}_{2}\right), 7.41(\mathrm{~s}, 1 \mathrm{H}), 7.48(\mathrm{~m}, 3 \mathrm{H}, \mathrm{Ph}), 8.35$ (s, 1H), 8.46 (m, 2H, Ph); $\left.{ }^{13} \mathrm{C} \mathrm{NMR} \mathrm{(100.62} \mathrm{MHz,} \mathrm{CDCl}_{3}\right) \delta 22.7$, $33.5,113.7$ (q, $\left.J_{\mathrm{CF}} 3.0 \mathrm{~Hz}\right), 120.6$ (q, $\left.J_{\mathrm{CF}} 276 \mathrm{~Hz}\right), 128.5$, 128.56, 131.6, 136.0, 152.9, 156.0 (q, $\left.J_{\mathrm{CF}} 34 \mathrm{~Hz}\right), 165.1$, 165.8, 170.0; ${ }^{19} \mathrm{~F}$ NMR $\left(376.4 \mathrm{MHz}, \mathrm{CDCl}_{3}\right) \delta-72.7$; HRMS (FTMS + pESI) $m / z$, calcd. for $\mathrm{C}_{15} \mathrm{H}_{11} \mathrm{~F}_{3} \mathrm{~N}_{4} \mathrm{O}$ [ $\left.\mathrm{MH}^{+}\right]$: 321.0963, found: $321.0977\left[\mathrm{MH}^{+}\right]$. Anal. calcd. for $\mathrm{C}_{15} \mathrm{H}_{11} \mathrm{~F}_{3} \mathrm{~N}_{4} \mathrm{O} 320.27 \mathrm{~g} \mathrm{~mol}^{-1}$ : C, 56.25; H, 3.46; N, 17.49; found: C, 56.50; H, 3.50; N, 17.28.

2-[2-(2-Methylthio-6-trifluoromethylpyrimidin-4-yl)ethyl]1,3,4-oxadiazole (4c)

Obtained $(68 \%)$ as a yellowish wax; ${ }^{1} \mathrm{H}$ NMR $\left(400.13 \mathrm{MHz}, \mathrm{CDCl}_{3}\right) \delta 2.55\left(\mathrm{~s}, 3 \mathrm{H}, \mathrm{SCH}_{3}\right), 3.36(\mathrm{~m}$, $\left.2 \mathrm{H}, \mathrm{CH}_{2}\right), 3.43\left(\mathrm{~m}, 2 \mathrm{H}, \mathrm{CH}_{2}\right), 7.18(\mathrm{~s}, 1 \mathrm{H}), 8.35$ (s, 1H); ${ }^{13} \mathrm{C} \mathrm{NMR}\left(100.62 \mathrm{MHz}, \mathrm{CDCl}_{3}\right) \delta 14.0,22.6,33.3,111.7$ $\left(\mathrm{q}, J_{\mathrm{CF}} 2.4 \mathrm{~Hz}\right), 120.2\left(\mathrm{q}, J_{\mathrm{CF}} 276 \mathrm{~Hz}\right), 153.9,155.7$ (q, $\left.J_{\mathrm{CF}} 36 \mathrm{~Hz}\right), 165.6,170.0,174.3 ;{ }^{19} \mathrm{~F}$ NMR $(376.4 \mathrm{MHz}$, $\left.\mathrm{CDCl}_{3}\right) \delta-71.7$; HRMS (FTMS + pESI) $\mathrm{m} / z$, calcd. for 
$\mathrm{C}_{10} \mathrm{H}_{9} \mathrm{~F}_{3} \mathrm{~N}_{4} \mathrm{OS}\left[\mathrm{MH}^{+}\right]: 291.0527$, found: $291.0519\left[\mathrm{MH}^{+}\right]$. Anal. calcd. for $\mathrm{C}_{10} \mathrm{H}_{9} \mathrm{~F}_{3} \mathrm{~N}_{4} \mathrm{OS} 290.26 \mathrm{~g} \mathrm{~mol}^{-1}: \mathrm{C}, 41.38 ; \mathrm{H}$, 3.13; N, 19.29; found: C, 41.20; H, 3.10; N, 19.15 .

2-[2-(2-Methyl-7-trifluoromethylpyrazolo[1,5-a]pyrimidin5-yl)ethyl]-1,3,4-oxadiazole (4d)

Obtained (65\%) as a white wax; ${ }^{1} \mathrm{H}$ NMR $(400.13 \mathrm{MHz}$, DMSO- $\left.d_{6}\right) \delta 2.45\left(\mathrm{~s}, 3 \mathrm{H}, \mathrm{CH}_{3}\right), 3.40\left(\mathrm{~m}, 4 \mathrm{H}, \mathrm{CH}_{2}\right), 6.64$ (s, 1H), $7.56(\mathrm{~s}, 1 \mathrm{H}), 9.08(\mathrm{~s}, 1 \mathrm{H}) ;{ }^{13} \mathrm{C} \mathrm{NMR}(100.62 \mathrm{MHz}$, DMSO- $\left.d_{6}\right) \delta 14.0,22.0,33.1,96.3,106.6\left(\mathrm{q}, J_{\mathrm{CF}} 3.0 \mathrm{~Hz}\right)$, $119.4\left(\mathrm{q}, J_{\mathrm{CF}} 275 \mathrm{~Hz}\right), 131.4\left(\mathrm{q}, J_{\mathrm{CF}} 38 \mathrm{~Hz}\right), 149.1,154.1$, $155.3,159.4,165.3 ;{ }^{19} \mathrm{~F}$ NMR (376.4 MHz, DMSO- $d_{6}$ ) $\delta-72.3$; HRMS (FTMS + pESI) $m / z$, calcd. for $\mathrm{C}_{12} \mathrm{H}_{10} \mathrm{~F}_{3} \mathrm{~N}_{5} \mathrm{O}$ [ $\left.\mathrm{MH}^{+}\right]$: 298.0915, found: $298.0839\left[\mathrm{MH}^{+}\right]$. Anal. calcd. for $\mathrm{C}_{12} \mathrm{H}_{10} \mathrm{~F}_{3} \mathrm{~N}_{5} \mathrm{O} 297.23 \mathrm{~g} \mathrm{~mol}^{-1}$ : C, 48.49; H, 3.39; N, 23.56; found: C, 48.52; H, 3.41; N, 23.50.

2-Methyl-5-[2-(5-trifluoromethyl-1 H-pyrazol-3-yl)ethyl]1,3,4-oxadiazole (5a)

Obtained (97\%) as a white wax; ${ }^{1} \mathrm{H}$ NMR $(400.13 \mathrm{MHz}$, $\left.\mathrm{CDCl}_{3}\right) \delta 2.50\left(\mathrm{~s}, 3 \mathrm{H}, \mathrm{CH}_{3}\right), 3.16\left(\mathrm{~m}, 2 \mathrm{H}, \mathrm{CH}_{2}\right), 3.25(\mathrm{~m}$, $\left.2 \mathrm{H}, \mathrm{CH}_{2}\right), 6.37$ (s, $\left.1 \mathrm{H}\right) ;{ }^{13} \mathrm{C}$ NMR (100.62 MHz, $\mathrm{CDCl}_{3}$ ) $\delta 10.7,21.7,25.2,102.5,121.5\left(\mathrm{q}, J_{\mathrm{CF}} 272 \mathrm{~Hz}\right), 142.9,143.1$ (q, $\left.J_{\mathrm{CF}} 36 \mathrm{~Hz}\right), 164.2,166.1 ;{ }^{19} \mathrm{~F} \mathrm{NMR}\left(376.4 \mathrm{MHz}, \mathrm{CDCl}_{3}\right)$ $\delta-61.7 ;$ HRMS (FTMS + pESI) $m / z$, calcd. for $\mathrm{C}_{9} \mathrm{H}_{9} \mathrm{~F}_{3} \mathrm{~N}_{4} \mathrm{O}$ $\left[\mathrm{MH}^{+}\right]$: 247.0807, found: $247.0799\left[\mathrm{MH}^{+}\right]$. Anal. calcd. for $\mathrm{C}_{9} \mathrm{H}_{9} \mathrm{~F}_{3} \mathrm{~N}_{4} \mathrm{O} 246.19 \mathrm{~g} \mathrm{~mol}^{-1}: \mathrm{C}, 43.91 ; \mathrm{H}, 3.68 ; \mathrm{N}, 22.76$; found: C, 44.00; H, 3.65; N, 22.80 .

2-Methyl-5-[2-(2-phenyl-6-trifluoromethylpyrimidin-4-yl) ethyl]-1,3,4-oxadiazole (5b)

Obtained $(81 \%)$ as a white solid, mp $129-130{ }^{\circ} \mathrm{C}$; ${ }^{1} \mathrm{HNMR}\left(400.13 \mathrm{MHz}, \mathrm{CDCl}_{3}\right) \delta 2.47\left(\mathrm{~s}, 3 \mathrm{H}, \mathrm{CH}_{3}\right), 3.44$ (m, $\left.4 \mathrm{H}, \mathrm{CH}_{2}\right), 7.41$ (s, 1H), 7.49 (m, 3H, Ph), 8.47 (m, 2H, Ph); ${ }^{13} \mathrm{C}$ NMR (100.62 MHz, $\left.\mathrm{CDCl}_{3}\right) \delta 10.7,23.0,33.7,113.7$ $\left(\mathrm{q}, J_{\mathrm{CF}} 3.0 \mathrm{~Hz}\right), 120.6\left(\mathrm{q}, J_{\mathrm{CF}} 276 \mathrm{~Hz}\right), 128.5,131.5,136.1$, $156.0\left(\mathrm{q}, J_{\mathrm{CF}} 34 \mathrm{~Hz}\right), 163.7,165.1,165.7,170.2 ;{ }^{19} \mathrm{~F} \mathrm{NMR}$ $\left(376.4 \mathrm{MHz}, \mathrm{CDCl}_{3}\right) \delta-72.7$; HRMS (FTMS + pESI) $\mathrm{m} / z$, calcd. for $\mathrm{C}_{16} \mathrm{H}_{13} \mathrm{~F}_{3} \mathrm{~N}_{4} \mathrm{O}\left[\mathrm{MH}^{+}\right]$: 335.1119 , found: 335.1151 $\left[\mathrm{MH}^{+}\right]$. Anal. calcd. $\mathrm{C}_{16} \mathrm{H}_{12} \mathrm{~F}_{3} \mathrm{~N}_{4 \mathrm{O}} 334.29 \mathrm{~g} \mathrm{~mol}^{-1}$ : C, 57.49; H, 3.92; N, 16.76; found: C, 57.60; H, 4.00; N, 16.80 .

2-Methyl-5-[2-(2-methylthio-6-trifluoromethylpyrimidin-4-yl) ethyl]-1,3,4-oxadiazole (5c)

Obtained (92\%) as a white wax; ${ }^{1} \mathrm{H}$ NMR $(400.13 \mathrm{MHz}$, $\left.\mathrm{CDCl}_{3}\right) \delta 2.48\left(\mathrm{~s}, 3 \mathrm{H}, \mathrm{CH}_{3}\right), 2.56\left(\mathrm{~s}, 3 \mathrm{H}, \mathrm{SCH}_{3}\right), 3.27(\mathrm{~m}$, $\left.4 \mathrm{H}, \mathrm{CH}_{2}\right), 7.17(\mathrm{~s}, 1 \mathrm{H}) ;{ }^{13} \mathrm{C}$ NMR $\left(100.62 \mathrm{MHz}, \mathrm{CDCl}_{3}\right)$ $\delta 10.8,14.1,22.9,33.4,111.1\left(\mathrm{q}, J_{\mathrm{CF}} 3.0 \mathrm{~Hz}\right), 120.3(\mathrm{q}$, $\left.J_{\mathrm{CF}} 276 \mathrm{~Hz}\right), 155.7\left(\mathrm{q}, J_{\mathrm{CF}} 34 \mathrm{~Hz}\right), 163.8,165.5,170.3$, 174.3; ${ }^{19} \mathrm{~F}$ NMR (376.4 MHz, $\mathrm{CDCl}_{3}$ ) $\delta-71.7$; HRMS (FTMS + pESI) $m / z$, calcd. for $\mathrm{C}_{11} \mathrm{H}_{11} \mathrm{~F}_{3} \mathrm{~N}_{4} \mathrm{OS}\left[\mathrm{MH}^{+}\right]$:
304.0684, found: 305.0683. Anal. calcd. for $\mathrm{C}_{11} \mathrm{H}_{11} \mathrm{~F}_{3} \mathrm{~N}_{4} \mathrm{OS}$ $304.29 \mathrm{~g} \mathrm{~mol}^{-1}$ : C, 43.42; H, 3.64; N, 18.40; found: C, 43.50; $\mathrm{H}, 3.70 ; \mathrm{N}, 18.55$.

2-Methyl-5-[2-(2-methyl-7-trifluoromethylpyrazolo [1,5-a]pyrimidin-5-yl)ethyl]-1,3,4-oxadiazole (5d)

Obtained (65\%) as a white wax; ${ }^{1} \mathrm{H}$ NMR $(400.13 \mathrm{MHz}$, DMSO- $\left.d_{6}\right) \delta 2.42\left(\mathrm{~s}, 3 \mathrm{H}, \mathrm{CH}_{3}\right), 2.44\left(\mathrm{~s}, 3 \mathrm{H}, \mathrm{CH}_{3}\right), 3.33(\mathrm{~m}$, $\left.4 \mathrm{H}, \mathrm{CH}_{2}\right), 6.64(\mathrm{~s}, 1 \mathrm{H}), 7.55(\mathrm{~s}, 1 \mathrm{H}) ;{ }^{13} \mathrm{C} \mathrm{NMR}(100.62 \mathrm{MHz}$, DMSO- $\left.d_{6}\right) \delta 10.3,14.0,22.2,33.1,96.4,106.8(\mathrm{q}$, $\left.J_{\mathrm{CF}} 3.0 \mathrm{~Hz}\right), 119.5\left(\mathrm{q}, J_{\mathrm{CF}} 274 \mathrm{~Hz}\right), 131.4\left(\mathrm{q}, J_{\mathrm{CF}} 38 \mathrm{~Hz}\right)$, 149.1, 155.4, 159.6, 163.4, 165.5; ${ }^{19} \mathrm{~F}$ NMR $(376.4 \mathrm{MHz}$, DMSO- $d_{6}$ ) $\delta-72.3$; HRMS (FTMS + pESI) $\mathrm{m} / z$, calcd. for $\mathrm{C}_{13} \mathrm{H}_{12} \mathrm{~F}_{3} \mathrm{~N}_{5} \mathrm{O}\left[\mathrm{MH}^{+}\right]$: 312.1072 , found: 312.1068 $\left[\mathrm{MH}^{+}\right]$. Anal. calcd. for $\mathrm{C}_{13} \mathrm{H}_{12} \mathrm{~F}_{3} \mathrm{~N}_{5} \mathrm{O} 311.26 \mathrm{~g} \mathrm{~mol}^{-1}$ : C, 50.16; H, 3.89; N, 22.50; found: C, 50.20; H, 3.91; N, 22.55 .

2-Phenyl-5-[2-(5-trifluoromethyl-1H-pyrazol-3-yl)ethyl]1,3,4-oxadiazole (6a)

Obtained $(78 \%)$ as a white solid, mp $173-175{ }^{\circ} \mathrm{C}$; ${ }^{1} \mathrm{H}$ NMR (400.13 MHz, DMSO- $\left.d_{6}\right) \delta 3.22(\mathrm{~m}, 2 \mathrm{H}$, $\left.\mathrm{CH}_{2}\right), 3.34\left(\mathrm{~m}, 2 \mathrm{H}, \mathrm{CH}_{2}\right), 6.56(\mathrm{~s}, 1 \mathrm{H}), 7.59(\mathrm{~m}, 3 \mathrm{H}, \mathrm{Ph})$, $7.95(\mathrm{~m}, 2 \mathrm{H}, \mathrm{Ph}), 13.4(\mathrm{NH}) ;{ }^{13} \mathrm{C}$ NMR $(100.62 \mathrm{MHz}$, DMSO- $\left.d_{6}\right) \delta 22.3,25.0,102.3,122.3\left(\mathrm{q}, J_{\mathrm{CF}} 270 \mathrm{~Hz}\right)$, $124,126.8,129.7,132.1,141.7,143.7$ (q, $\left.J_{\mathrm{CF}} 36 \mathrm{~Hz}\right)$, 164.6, 166.1; ${ }^{19} \mathrm{~F}$ NMR (376.4 MHz, DMSO- $d_{6}$ ) $\delta-61.8$; HRMS (FTMS + pESI) $\mathrm{m} / z$, calcd. for $\mathrm{C}_{14} \mathrm{H}_{11} \mathrm{~F}_{3} \mathrm{~N}_{4} \mathrm{O}$ [ $\left.\mathrm{MH}^{+}\right]$: 309.0963, found: $309.0971\left[\mathrm{MH}^{+}\right]$. Anal. calcd. for $\mathrm{C}_{14} \mathrm{H}_{11} \mathrm{~F}_{3} \mathrm{~N}_{4} \mathrm{O} 308.26 \mathrm{~g} \mathrm{~mol}^{-1}$ : C, 54.55; H, 3.60; N, 18.18; found: $\mathrm{C}, 54.60 ; \mathrm{H}, 3.60 ; \mathrm{N}, 18.22$.

2-Phenyl-5-[2-(2-phenyl-6-trifluoromethylpyrimidin-4-yl) ethyl]-1,3,4-oxadiazole (6b)

Obtained (98\%) as yellow solid, mp $116-117{ }^{\circ} \mathrm{C}$; ${ }^{1} \mathrm{H}$ NMR (400.13 MHz, $\left.\mathrm{CDCl}_{3}\right) \delta 3.52\left(\mathrm{~s}, 2 \mathrm{H}, \mathrm{CH}_{2}\right)$, $3.58\left(\mathrm{~m}, 2 \mathrm{H}, \mathrm{CH}_{2}\right), 7.48(\mathrm{~s}, 1 \mathrm{H}), 7.49(\mathrm{~m}, 6 \mathrm{H}, \mathrm{Ph}), 7.99$ $(\mathrm{m}, 2 \mathrm{H}, \mathrm{Ph}), 8.46$ (m, 2H, Ph); ${ }^{13} \mathrm{C}$ NMR (100.62 MHz, $\left.\mathrm{CDCl}_{3}\right) \delta 23.2,33.8,113.8\left(\mathrm{q}, J_{\mathrm{CF}} 3.0 \mathrm{~Hz}\right), 120.7(\mathrm{q}$, $\left.J_{\mathrm{CF}} 275 \mathrm{~Hz}\right), 123.8,126.7,128.6,129.0,131.6,136.1$, $156.1\left(\mathrm{q}, J_{\mathrm{CF}} 34 \mathrm{~Hz}\right), 164.9,165.2,165.6,170.2 ;{ }^{19} \mathrm{~F}$ NMR $\left(376.4 \mathrm{MHz}, \mathrm{CDCl}_{3}\right) \delta-72.7$; HRMS (FTMS + pESI) $\mathrm{m} / z$, calcd. for $\mathrm{C}_{21} \mathrm{H}_{15} \mathrm{~F}_{3} \mathrm{~N}_{4} \mathrm{O}\left[\mathrm{MH}^{+}\right]$: 397.1276, found: $\left[\mathrm{MH}^{+}\right]$ 397.1283. Anal. calcd. for $\mathrm{C}_{21} \mathrm{H}_{15} \mathrm{~F}_{3} \mathrm{~N}_{4} \mathrm{O} 396.36 \mathrm{~g} \mathrm{~mol}^{-1}$ : C, $63.63 ; \mathrm{H}, 3.81 ; \mathrm{N}, 14.15$; found: C, 63.60; H, 3.85; N, 14.14 .

2-[2-(2-Methylthio-6-trifluoromethylpyrimidin-4-yl) ethyl]-5-phenyl-1,3,4-oxadiazole (6c)

Obtained $(78 \%)$ as a white solid, mp $141-142{ }^{\circ} \mathrm{C}$; ${ }^{1} \mathrm{H}$ NMR (400.13 MHz, $\left.\mathrm{CDCl}_{3}\right) \delta 2.55\left(\mathrm{~s}, 3 \mathrm{H}, \mathrm{SCH}_{3}\right.$ ), $3.41\left(\mathrm{~m}, 2 \mathrm{H}, \mathrm{CH}_{2}\right), 3.47\left(\mathrm{~m}, 2 \mathrm{H}, \mathrm{CH}_{2}\right), 7.22(\mathrm{~s}, 1 \mathrm{H}), 7.51$ 
(m, 3H, Ph), 7.99 (m, 2H, Ph); ${ }^{13} \mathrm{C}$ NMR (100.62 MHz, $\left.\mathrm{CDCl}_{3}\right) \delta 14.0,23.0,33.4,111.1\left(\mathrm{q}, J_{\mathrm{CF}} 3.0 \mathrm{~Hz}\right), 120.2$ $\left(\mathrm{q}, J_{\mathrm{CF}} 276 \mathrm{~Hz}\right), 126.7,129.0,131.6,155.6\left(\mathrm{q}, J_{\mathrm{CF}} 36 \mathrm{~Hz}\right)$, $164.8,165.3,170.2,174.2 ;{ }^{19} \mathrm{~F}$ NMR $(376.4 \mathrm{MHz}$, $\left.\mathrm{CDCl}_{3}\right) \delta-71.7$; HRMS (FTMS + pESI) $\mathrm{m} / z$, calcd. for $\mathrm{C}_{16} \mathrm{H}_{13} \mathrm{~F}_{3} \mathrm{~N}_{4} \mathrm{OS}\left[\mathrm{MH}^{+}\right]: 367.0841$, found: [ $\left.\mathrm{MH}^{+}\right] 367.0854$. Anal. calcd. for $\mathrm{C}_{16} \mathrm{H}_{13} \mathrm{~F}_{3} \mathrm{~N}_{4} \mathrm{OS} 366.36 \mathrm{~g} \mathrm{~mol}^{-1}: \mathrm{C}, 43.42 ; \mathrm{H}$, 3.64; N, 18.40; found: C, 43.50; H, 3.70; N, 18.55 .

2-[2-(2-Methyl-7-trifluoromethylpyrazolo[1,5-a]pyrimidin5-yl)ethyl]-5-phenyl-1,3,4-oxadiazole (6d)

Obtained $(87 \%)$ as a yellow solid, mp $158-160{ }^{\circ} \mathrm{C}$; ${ }^{1} \mathrm{H}$ NMR (400.13 MHz, DMSO- $\left.d_{6}\right) \delta 2.45\left(\mathrm{~s}, 3 \mathrm{H}, \mathrm{CH}_{3}\right)$, $3.48\left(\mathrm{~m}, 4 \mathrm{H}, \mathrm{CH}_{2}\right), 6.65(\mathrm{~s}, 1 \mathrm{H}), 7.56(\mathrm{~s}, 1 \mathrm{H}), 7.58(\mathrm{~m}, 3 \mathrm{H}$, $\mathrm{Ph}), 7.93$ (m, 2H, Ph); ${ }^{13} \mathrm{C}$ NMR (100.62 MHz, DMSO- $d_{6}$ ) $\delta 13.9,22.4,33.1,96.2,106.8\left(\mathrm{q}, J_{\mathrm{CF}} 4.0 \mathrm{~Hz}\right), 119.5(\mathrm{q}$, $\left.J_{\mathrm{CF}} 275 \mathrm{~Hz}\right), 123.4,126.1,129.0,131.4\left(\mathrm{q}, J_{\mathrm{CF}} 38 \mathrm{~Hz}\right), 131.5$, 149.1, 155.2, 159.4, 163.7, 165.7; ${ }^{19} \mathrm{~F}$ NMR $(376.4 \mathrm{MHz}$, DMSO- $\left.d_{6}\right) \delta-72.3$. HRMS (FTMS + pESI) $\mathrm{m} / z$, calcd. for $\mathrm{C}_{13} \mathrm{H}_{12} \mathrm{~F}_{3} \mathrm{~N}_{5} \mathrm{O}\left[\mathrm{MH}^{+}\right]$: 373.1228, found: $374.1188\left[\mathrm{MH}^{+}\right]$. Anal. calcd. for $\mathrm{C}_{13} \mathrm{H}_{12} \mathrm{~F}_{3} \mathrm{~N}_{5} \mathrm{O} 373.33 \mathrm{~g} \mathrm{~mol}^{-1}$ : C, 50.16; H, $3.89 ; \mathrm{N}, 22.50$; found: C, 50.20; H, 3.91; N, 22.53 .

General procedure for the synthesis of 5-substituted1,3,4-oxadiazole-2-thiol (7a-d)

Carbon disulfide $(2 \mathrm{~mL})$ was added dropwise to a solution of the prepared hydrazide 3a-d $(5 \mathrm{mmol})$ in ethanolic potassium hydroxide $(0.3 \mathrm{~g} / 10 \mathrm{~mL})$, under stirring over a period of $30 \mathrm{~min}$. Stirring was continued for another $30 \mathrm{~min}$. The reaction mixture was heated to $50{ }^{\circ} \mathrm{C}$ until the evolution of all $\mathrm{H}_{2} \mathrm{~S}$ ceased. The salt that formed was dissolved in water and acidified with $\mathrm{HCl}$; the mass obtained was filtered off to give the following:

5-[2-(5-Trifluoromethyl-1 H-pyrazol-3-yl)ethyl]1,3,4-oxadiazole-2-thiol (7a)

Obtained (69\%) as an off-white solid; ${ }^{1} \mathrm{H}$ NMR $\left(400.13 \mathrm{MHz}, \mathrm{CDCl}_{3}\right) \delta 3.11\left(\mathrm{~m}, 4 \mathrm{H}, \mathrm{CH}_{2}\right), 6.53(\mathrm{~s}, 1 \mathrm{H})$, 13.4 (br s, $1 \mathrm{H}, \mathrm{NH}) ;{ }^{13} \mathrm{C} \mathrm{NMR}\left(100.62 \mathrm{MHz}, \mathrm{CDCl}_{3}\right) \delta 20.8$, $24.4,101.9,121.8$ (q, $\left.J_{\mathrm{CF}} 270 \mathrm{~Hz}\right), 141.1$ (q, $J_{\mathrm{CF}} 36 \mathrm{~Hz}$ ), 143.0, 162.8, 177.8; ${ }^{19} \mathrm{~F}$ NMR $\left(376.4 \mathrm{MHz}, \mathrm{CDCl}_{3}\right) \delta-62.1$; HRMS (FTMS + pESI) $\mathrm{m} / z$, calcd. for $\mathrm{C}_{8} \mathrm{H}_{7} \mathrm{~F}_{3} \mathrm{~N}_{4} \mathrm{OS}$ $\left[\mathrm{M}^{+}\right]$: 265.0370, found: $265.0371\left[\mathrm{MH}^{+}\right]$. Anal. calcd. for $\mathrm{C}_{8} \mathrm{H}_{7} \mathrm{~F}_{3} \mathrm{~N}_{4} \mathrm{OS} 264.23 \mathrm{~g} \mathrm{~mol}^{-1}$ : C, 36.36; H, 2.67; N, 21.20; found: C, 36.31; H, 2.70; N, 21.17.

5-[2-(2-Phenyl-6-trifluoromethylpyrimidin-4-yl)ethyl]1,3,4-oxadiazole-2-thiol (7b)

Obtained (90\%) as a yellow solid, mp $163-164{ }^{\circ} \mathrm{C}$; ${ }^{1} \mathrm{H}$ NMR (400.13 MHz, $\left.\mathrm{CDCl}_{3}\right) \delta 3.35\left(\mathrm{~m}, 4 \mathrm{H}, \mathrm{CH}_{2}\right.$ ), $7.38(\mathrm{~s}, 1 \mathrm{H}), 7.49(\mathrm{~m}, 3 \mathrm{H}, \mathrm{Ph}), 8.45(\mathrm{~m}, 2 \mathrm{H}, \mathrm{Ph})$; ${ }^{13} \mathrm{C}$ NMR (100.62 MHz, $\left.\mathrm{CDCl}_{3}\right) \delta 23.3,32.6,113.6(\mathrm{q}$, $\left.J_{\mathrm{CF}} 2.7 \mathrm{~Hz}\right), 120.6\left(\mathrm{q}, J_{\mathrm{CF}} 275 \mathrm{~Hz}\right), 128.7,131.8,136.0$, $156.3\left(\mathrm{q}, J_{\mathrm{CF}} 36 \mathrm{~Hz}\right), 163.4,165.4,169.3,178.7 ;{ }^{19} \mathrm{~F}$ NMR $\left(376.4 \mathrm{MHz}, \mathrm{CDCl}_{3}\right) \delta-72.7$; HRMS (FTMS + pESI) $\mathrm{m} / z$, calcd. for $\mathrm{C}_{15} \mathrm{H}_{11} \mathrm{~F}_{3} \mathrm{~N}_{4} \mathrm{OS}\left[\mathrm{MH}^{+}\right]: 353.0683$, found: 353.0691 $\left[\mathrm{MH}^{+}\right]$. Anal. calcd. for $\mathrm{C}_{15} \mathrm{H}_{11} \mathrm{~F}_{3} \mathrm{~N}_{4} \mathrm{OS} 352.33 \mathrm{~g} \mathrm{~mol}^{-1}$ : C, 50.99; H, 3.42; N, 15.86; found: C, 51.05; H, 3.45; N, 15.90 .

5-[2-(2-Methylthio-6-trifluoromethylpyrimidin-4-yl)ethyl]1,3,4-oxadiazole-2-thiol (7c)

Obtained (84\%) as a yellow solid, mp $156-158{ }^{\circ} \mathrm{C}$; ${ }^{1} \mathrm{H}$ NMR $\left(400.13 \mathrm{MHz}, \mathrm{CDCl}_{3}\right) \delta 2.56\left(\mathrm{~s}, 3 \mathrm{H}, \mathrm{SCH}_{3}\right)$, $3.25\left(\mathrm{~m}, 4 \mathrm{H}, \mathrm{CH}_{2}\right), 7.14(\mathrm{~s}, 1 \mathrm{H}), 8.35(\mathrm{~s}, 1 \mathrm{H}) ;{ }^{13} \mathrm{C} \mathrm{NMR}$ $\left(100.62 \mathrm{MHz}, \mathrm{CDCl}_{3}\right) \delta 14.2,23.2,32.4,110.9$ (q, $\left.J_{\mathrm{CF}} 2.7 \mathrm{~Hz}\right), 120.3\left(\mathrm{q}, J_{\mathrm{CF}} 276 \mathrm{~Hz}\right), 155.9\left(\mathrm{q}, J_{\mathrm{CF}} 36 \mathrm{~Hz}\right)$, 163.1, 169.3, 174.7, 178.7; ${ }^{19} \mathrm{~F}$ NMR $(376.4 \mathrm{MHz}$, $\left.\mathrm{CDCl}_{3}\right) \delta-71.5$; HRMS (FTMS + pESI) $\mathrm{m} / z$, calcd. for $\mathrm{C}_{10} \mathrm{H}_{9} \mathrm{~F}_{3} \mathrm{~N}_{4} \mathrm{OS}_{2}\left[\mathrm{MH}^{+}\right]$: 323.0248 , found: $323.0165\left[\mathrm{MH}^{+}\right]$. Anal. Calcd. for $\mathrm{C}_{10} \mathrm{H}_{9} \mathrm{~F}_{3} \mathrm{~N}_{4} \mathrm{OS}_{2} 322.33 \mathrm{~g} \mathrm{~mol}^{-1}$ : C, 37.26; H, 2.81; N, 17.38; found: C, 37.20; H, 2.80; N, 17.35 .

5-[2-(2-Methyl-7-trifluoromethylpyrazolo[1,5-a]pyrimidin5-yl)ethyl]-1,3,4-oxadiazole-2-thiol (7d)

Obtained (97\%) as a yellow solid, decomposes upper $200{ }^{\circ} \mathrm{C}$; ${ }^{1} \mathrm{H}$ NMR $\left(400.13 \mathrm{MHz}\right.$, DMSO- $\left.d_{6}\right) \delta 2.46$ (s, 3H, $\left.\mathrm{CH}_{3}\right), 3.26\left(\mathrm{t}, 2 \mathrm{H}, \mathrm{CH}_{2}\right), 3.33\left(\mathrm{t}, 2 \mathrm{H}, \mathrm{CH}_{2}\right), 6.66(\mathrm{~s}, 1 \mathrm{H}), 7.55$ (s, 1H), 14.2 (s, SH); ${ }^{13} \mathrm{C}$ NMR (100.62 MHz, DMSO- $d_{6}$ ) $\delta 14.0,22.5,32.2,96.4,106.6\left(\mathrm{q}, J_{\mathrm{CF}} 2.5 \mathrm{~Hz}\right), 119.4(\mathrm{q}$, $\left.J_{\mathrm{CF}} 275 \mathrm{~Hz}\right), 131.4\left(\mathrm{q}, J_{\mathrm{CF}} 36 \mathrm{~Hz}\right), 149.0,155.3,159.2$, 163.2, 177.6; ${ }^{19} \mathrm{~F}$ NMR (376.4 MHz, DMSO- $d_{6}$ ) $\delta-72.5$; HRMS (FTMS + pESI) $\mathrm{m} / z$, calcd. for $\mathrm{C}_{12} \mathrm{H}_{10} \mathrm{~F}_{3} \mathrm{~N}_{5} \mathrm{OS}$ [ $\left.\mathrm{MH}^{+}\right]$: 330.0636, found: $330.0702\left[\mathrm{MH}^{+}\right]$. Anal. calcd. for $\mathrm{C}_{12} \mathrm{H}_{10} \mathrm{~F}_{3} \mathrm{~N}_{5} \mathrm{OS} 329.30 \mathrm{~g} \mathrm{~mol}^{-1}$ : C, 48.49; H, 3.39; N, 23.56; found: C, 48.52; H, 3.41; N, 23.50.

General procedure for the $S$-alkylation of 5 -substituted1,3,4-oxadiazole-2-thiol with 2-bromoacetophenone. Synthesis of $\mathbf{8 b}$ and $\mathbf{8 c}$

A solution of 2-bromoacetophenone ( $3 \mathrm{mmol}$ ) in $\mathrm{CHCl}_{3}$ $\left(5 \mathrm{~mL}\right.$ ) was added dropwise to a stirred and cooled (to $0{ }^{\circ} \mathrm{C}$ ) solution of the respective compound $7(3 \mathrm{mmol})$ and $\mathrm{Et}_{3} \mathrm{~N}$ ( $3 \mathrm{mmol})$ in $\mathrm{CHCl}_{3}(15 \mathrm{~mL})$. The reaction mixture was stirred for $1 \mathrm{~h}$ and then was poured into water. The organic layer was separated and washed with water $(10 \mathrm{~mL} \times 2)$. The organic layer was dried over $\mathrm{Na}_{2} \mathrm{SO}_{4}$, and then the solvent was removed under vacuum and the solid products were identified. A portion was recrystallized from $\mathrm{CHCl}_{3} /$ hexane solutions for elemental analysis experiments. 
1-Phenyl-2-[(5-(2-(2-phenyl-6-trifluoromethylpyrimidin-4-yl) ethyl)-1,3,4-oxadiazol-2-yl)thio]ethanone (8b)

Obtained $(86 \%)$ as a white solid, $\mathrm{mp} 155-156{ }^{\circ} \mathrm{C}$; ${ }^{1} \mathrm{H}$ NMR (400.13 MHz, $\left.\mathrm{CDCl}_{3}\right) \delta 3.47\left(\mathrm{~m}, 4 \mathrm{H}, 2 \mathrm{CH}_{2}\right)$, $4.42\left(\mathrm{~s}, 2 \mathrm{H}, \mathrm{SCH}_{2}\right), 7.42(\mathrm{~s}, 1 \mathrm{H}), 7.49(\mathrm{~m}, 5 \mathrm{H}, \mathrm{Ph})$, 7.62 (m, 1H, Ph), 7.99 (m, 2H, Ph), 8.47 (m, 2H, Ph); ${ }^{13} \mathrm{C}$ NMR (100.62 MHz, $\left.\mathrm{CDCl}_{3}\right) \delta 23.1,33.6,41.4,113.8$ $\left(\mathrm{q}, J_{\mathrm{CF}} 2.7 \mathrm{~Hz}\right), 120.7\left(\mathrm{q}, J_{\mathrm{CF}} 275 \mathrm{~Hz}\right), 128.4,128.6,128.9$, $131.7,134.2,134.9,136.1(\mathrm{Ph}), 156.1\left(\mathrm{q}, J_{\mathrm{CF}} 36 \mathrm{~Hz}\right.$ C-6 pym), 164.0 (C-2 pym), 165.3 (C-5, oxz), 167.0, 170.0, 192.0; ${ }^{19} \mathrm{~F}$ NMR (376.4 MHz, $\left.\mathrm{CDCl}_{3}\right) \delta-71.9$; HRMS (FTMS + pESI) $m / z$, calcd. for $\mathrm{C}_{23} \mathrm{H}_{17} \mathrm{~F}_{3} \mathrm{~N}_{4} \mathrm{O}_{2} \mathrm{~S}\left[\mathrm{MH}^{+}\right]$: 471.1102 found: $471.1097\left[\mathrm{MH}^{+}\right]$. Anal. calcd. for $\mathrm{C}_{23} \mathrm{H}_{17} \mathrm{~F}_{3} \mathrm{~N}_{4} \mathrm{O}_{2} \mathrm{~S} 470.46$ g mol$^{-1}$ : C, 58.72; H, 3.64; N, 11.91; found: C, 58.58; H, 3.60; N, 11.90 .

2-[(5-(2-(2-Methylthio-6-trifluoromethylpyrimidin-4-yl)ethyl)1,3,4-oxadiazol-2-yl)thio]-1-phenylethanone (enol form, 8c)

Obtained $(89 \%)$ as a white solid, decomposes upper $195{ }^{\circ} \mathrm{C} ;{ }^{1} \mathrm{H}$ NMR (400.13 MHz, $\left.\mathrm{CDCl}_{3}\right) \delta 2.53$ (s, 3H, $\left.\mathrm{SCH}_{3}\right), 2.77\left(\mathrm{t}, 2 \mathrm{H}, \mathrm{CH}_{2}\right), 3.02\left(\mathrm{t}, 2 \mathrm{H}, \mathrm{CH}_{2}\right), 6.07$ (s, 1H), 7.05 (s, 1H), 7.32 (m, 5H, Ph), 8.35 (br s, OH enol); ${ }^{13} \mathrm{C}$ NMR (100.62 MHz, $\left.\mathrm{CDCl}_{3}\right) \delta 14.1,30.7,32.1,96.5$, $111.3\left(\mathrm{q}, J_{\mathrm{CF}} 2.7 \mathrm{~Hz}\right), 120.3\left(\mathrm{q}, J_{\mathrm{CF}} 276 \mathrm{~Hz}\right), 128.2,128.4$, $129.5,137.4,155.5$ (q, $\left.J_{\mathrm{CF}} 36 \mathrm{~Hz}\right), 163.1,169.8,178.7$, 174.0; ${ }^{19} \mathrm{~F} \mathrm{NMR}\left(376.4 \mathrm{MHz}, \mathrm{CDCl}_{3}\right) \delta-71.5$. Anal. calcd. for $\mathrm{C}_{18} \mathrm{H}_{15} \mathrm{~F}_{3} \mathrm{~N}_{4} \mathrm{O}_{2} \mathrm{~S}_{2} 440.46 \mathrm{~g} \mathrm{~mol}^{-1}$ : C, 49.08; H, 3.43; N, 12.72; found: C, 49.10; H, 3.45; N, 12.65 .

General procedure for the $S$-acylation of 5-substituted1,3,4-oxadiazole-2-thiol with anhydride acetic and trichloroacetyl chloride. Synthesis of $\mathbf{9 b}-\mathbf{d}$ and $\mathbf{1 0 b}, \mathbf{c}$

A solution of acylating agent $\left(\mathrm{CH}_{3} \mathrm{CO}\right)_{2} \mathrm{O}$ or $\mathrm{Cl}_{3} \mathrm{CCOCl}$ ( $3 \mathrm{mmol}$ ) in $\mathrm{CHCl}_{3}(5 \mathrm{~mL})$ was added dropwise to a stirred and cooled (to $0{ }^{\circ} \mathrm{C}$ ) solution of the respective compound 7 ( $3 \mathrm{mmol}$ ) in $\mathrm{CHCl}_{3}(15 \mathrm{~mL})$. The reaction mixture was stirred for $1 \mathrm{~h}$, poured into water, and then the organic layer was separated and washed with water $(10 \mathrm{~mL} \times 4)$. The organic layer was dried over $\mathrm{Na}_{2} \mathrm{SO}_{4}$, the solvent was removed under vacuum, and the solid products were identified. A portion was recrystallized from $\mathrm{CHCl}_{3}$ / hexane solutions for elemental analysis experiments.

S-[5-(2-(2-Phenyl-6-trifluoromethylpyrimidin-4-yl)ethyl)1,3,4-oxadiazol-2-yl] ethanethioate $(\mathbf{9 b})$

Obtained $(92 \%)$ as a yellow solid, mp 142-144 ${ }^{\circ} \mathrm{C}$; ${ }^{1} \mathrm{H}$ NMR (400.13 MHz, $\left.\mathrm{CDCl}_{3}\right) \delta 2.58\left(\mathrm{~s}, 3 \mathrm{H}, \mathrm{CH}_{3}\right), 3.35$ (t, $\left.2 \mathrm{H}, \mathrm{CH}_{2}\right), 3.42\left(\mathrm{t}, 2 \mathrm{H}, \mathrm{CH}_{2}\right), 7.41(\mathrm{~s}, 1 \mathrm{H}), 7.49$ (m, 3H, Ph), 8.45 (m, 2H, Ph); $\left.{ }^{13} \mathrm{C} \mathrm{NMR} \mathrm{(100.62} \mathrm{MHz,} \mathrm{CDCl}_{3}\right) \delta 23.1$, 24.1, 32.3, $113.6\left(\mathrm{q}, J_{\mathrm{CF}} 2.7 \mathrm{~Hz}\right), 120.6\left(\mathrm{q}, J_{\mathrm{CF}} 275 \mathrm{~Hz}\right)$,
128.7, 131.8, 136.0, 156.3 (q, $\left.J_{\mathrm{CF}} 36 \mathrm{~Hz}\right), 163.4,165.3$, 166.1, 169.2, 173.7; ${ }^{19} \mathrm{~F}$ NMR (376.4 MHz, $\left.\mathrm{CDCl}_{3}\right) \delta$ -72.7. Anal. calcd. for $\mathrm{C}_{17} \mathrm{H}_{13} \mathrm{~F}_{3} \mathrm{~N}_{4} \mathrm{O}_{2} \mathrm{~S} 394.37 \mathrm{~g} \mathrm{~mol}^{-1}$ : C, 51.77; H, 3.32; N, 14.21; found: C, 51.75; H, 3.3; N, 14.15 .

5-[2-(2-Methylthio-6-trifluoromethylpyrimidin-4-yl)ethyl]1,3,4-oxadiazole-2-thiol (9c)

Obtained (98\%) as a white solid, mp $153-155{ }^{\circ} \mathrm{C}$; ${ }^{1} \mathrm{H}$ NMR (400.13 MHz, $\left.\mathrm{CDCl}_{3}\right) \delta 2.57$ (s, 3H, $\mathrm{SCH}_{3}$ ), 2.60 (s, 3H, $\left.\mathrm{CH}_{3}\right), 3.26\left(\mathrm{~m}, 2 \mathrm{H}, \mathrm{CH}_{2}\right), 3.33\left(\mathrm{~m}, 2 \mathrm{H}, \mathrm{CH}_{2}\right)$, $7.21(\mathrm{~s}, 1 \mathrm{H}) ;{ }^{13} \mathrm{C} \mathrm{NMR}\left(100.62 \mathrm{MHz}, \mathrm{CDCl}_{3}\right) \delta 13.9,22.7$, 24.1, 32.0, 110.9 (q, $\left.J_{\mathrm{CF}} 2.7 \mathrm{~Hz}\right), 120.1$ (q, $\left.J_{\mathrm{CF}} 276 \mathrm{~Hz}\right)$, 155.5 (q, $\left.J_{\mathrm{CF}} 36 \mathrm{~Hz}\right), 160.1,166.2,169.1,173.4,174.7$; ${ }^{19} \mathrm{~F}$ NMR $\left(376.4 \mathrm{MHz}, \mathrm{CDCl}_{3}\right) \delta-71.2$. Anal. calcd. for $\mathrm{C}_{12} \mathrm{H}_{11} \mathrm{~F}_{3} \mathrm{~N}_{4} \mathrm{O}_{2} \mathrm{~S}_{2} 364.03 \mathrm{~g} \mathrm{~mol}^{-1}: \mathrm{C}, 39.45 ; \mathrm{H}, 3.31 ; \mathrm{N}, 15.33$; found: C, 39.5; H, 3.3; N, 15.4.

S-[5-(2-(2-Methyl-7-trifluoromethylpyrazolo[1,5-a]pyrimidin5-yl)ethyl)-1,3,4-oxadiazol-2-yl] ethanethioate (9d)

Obtained $(88 \%)$ as a yellow solid, decomposes upper $210{ }^{\circ} \mathrm{C} ;{ }^{1} \mathrm{H}$ NMR (400.13 MHz, DMSO-d $\left.)_{6}\right) \quad 2.46$ $\left(\mathrm{s}, 3 \mathrm{H}, \mathrm{CH}_{3}\right), 2.64\left(\mathrm{~s}, 3 \mathrm{H}, \mathrm{CH}_{3}\right), 3.24\left(\mathrm{t}, 2 \mathrm{H}, \mathrm{CH}_{2}\right)$, $3.31\left(\mathrm{t}, 2 \mathrm{H}, \mathrm{CH}_{2}\right), 6.66(\mathrm{~s}, 1 \mathrm{H}), 7.65(\mathrm{~s}, 1 \mathrm{H}) ;{ }^{13} \mathrm{C} \mathrm{NMR}$ (100.62 MHz, DMSO- $\left.d_{6}\right) \delta 14.0,22.5,24.2,32.1,96.3$, $106.6\left(\mathrm{q}, J_{\mathrm{CF}} 2.5 \mathrm{~Hz}\right), 120.6\left(\mathrm{q}, J_{\mathrm{CF}} 275 \mathrm{~Hz}\right), 131.3(\mathrm{q}$, $\left.J_{\mathrm{CF}} 36 \mathrm{~Hz}\right), 149.0,155.2,159.0,163.1,174.7,177.6$; ${ }^{19} \mathrm{~F}$ NMR (376.4 MHz, DMSO- $\left.d_{6}\right) \delta-71.7$. Anal. calcd. for $\mathrm{C}_{14} \mathrm{H}_{12} \mathrm{~F}_{3} \mathrm{~N}_{5} \mathrm{O}_{2} \mathrm{~S} 371.32 \mathrm{~g} \mathrm{~mol}^{-1}$ : C, 45.28; H, 3.26; N, 18.86; found: C, 45.3; H, 3.25; N, 18.9.

S-[5-(2-(2-Phenyl-6-trifluoromethylpyrimidin-4-yl)ethyl)1,3,4-oxadiazol-2-yl] 2,2,2-trichloro ethanethioate (10b)

Obtained $(84 \%)$ as a brownish solid, mp $102-104{ }^{\circ} \mathrm{C}$; ${ }^{1} \mathrm{H}$ NMR (400.13 MHz, $\left.\mathrm{CDCl}_{3}\right) \delta 3.35\left(\mathrm{~m}, 4 \mathrm{H}, \mathrm{CH}_{2}\right)$, 7.37 (s, 1H, H5), 7.48 (m, 3H, Ph), 8.45 (m, 2H, Ph); ${ }^{13} \mathrm{C} \mathrm{NMR}\left(100.62 \mathrm{MHz}, \mathrm{CDCl}_{3}\right) \delta 23.2,32.6,91.5,113.7$ $\left(\mathrm{q}, J_{\mathrm{CF}} 2.7 \mathrm{~Hz}\right), 120.5\left(\mathrm{q}, J_{\mathrm{CF}} 275 \mathrm{~Hz}\right), 128.5,128.7,131.8$, $135.9,156.1$ (q, $\left.J_{\mathrm{CF}} 36 \mathrm{~Hz}\right), 163.3,165.2,169.3,176.8$, 178.6; ${ }^{19} \mathrm{~F} \mathrm{NMR}\left(376.4 \mathrm{MHz}, \mathrm{CDCl}_{3}\right) \delta-72.7$. Anal. calcd. for $\mathrm{C}_{17} \mathrm{H}_{10} \mathrm{Cl}_{3} \mathrm{~F}_{3} \mathrm{~N}_{4} \mathrm{O}_{2} \mathrm{~S} 497.70 \mathrm{~g} \mathrm{~mol}^{-1}$ : C, 51.77; H, 3.32; N, 14.21; found: C, 51.75; H, 3.3; N, 14.15.

S-[5-(2-(2-Methylthio-6-trifluoromethylpyrimidin-4-yl)ethyl)1,3,4-oxadiazol-2-yl] 2,2,2-trichloroethanethioate (10c)

Obtained (74\%) as a brownish solid, mp $138-139{ }^{\circ} \mathrm{C}$; ${ }^{1} \mathrm{H}$ NMR (400.13 MHz, $\left.\mathrm{CDCl}_{3}\right) \delta 2.57\left(\mathrm{~s}, 3 \mathrm{H}, \mathrm{SCH}_{3}\right), 3.28$ $\left(\mathrm{m}, 4 \mathrm{H}, 2 \mathrm{CH}_{2}\right), 7.16$ (s, 1H, H5); ${ }^{13} \mathrm{C} \mathrm{NMR}(100.62 \mathrm{MHz}$, $\left.\mathrm{CDCl}_{3}\right) \delta 14.2,23.3,32.5,90.0,111.1\left(\mathrm{q}, J_{\mathrm{CF}} 2.7 \mathrm{~Hz}\right), 120.3$ $\left(\mathrm{q}, J_{\mathrm{CF}} 276 \mathrm{~Hz}\right), 156.0\left(\mathrm{q}, J_{\mathrm{CF}} 36 \mathrm{~Hz}\right), 163.3,164.2,169.5$, 174.7, 178.8; ${ }^{19} \mathrm{~F}$ NMR $\left(376.4 \mathrm{MHz}, \mathrm{CDCl}_{3}\right) \delta-71.8$. Anal. 
calcd. for $\mathrm{C}_{12} \mathrm{H}_{8} \mathrm{Cl}_{3} \mathrm{~F}_{3} \mathrm{~N}_{4} \mathrm{O}_{2} \mathrm{~S}_{2} 467.70 \mathrm{~g} \mathrm{~mol}^{-1}$ : C, 30.82; H, 1.72 ; N, 11.98; found: C, 30.75; H, 1.75; N, 12.1 .

General procedure for the synthesis of $\mathrm{N}$-acylhydrazones (11b-d)

A solution of 4-chlorobenzaldehyde ( $0.43 \mathrm{~g}, 3 \mathrm{mmol})$ in EtOH $(2 \mathrm{~mL})$ was added to a stirred solution of the respective hydrazide $\mathbf{3 b}-\mathbf{d}(3 \mathrm{mmol})$ in $\mathrm{EtOH}(8 \mathrm{~mL})$. The reaction mixture was stirred at $50{ }^{\circ} \mathrm{C}$ for $6 \mathrm{~h}$. The solution was cooled at $0{ }^{\circ} \mathrm{C}$ until the product precipitated. Then the solid was filtered, washed with cooled $\mathrm{EtOH}$, and then dried under vacuum. The $\mathrm{N}$-acylhydrazones were solids and a portion was recrystallized from $\mathrm{CHCl}_{3}$ /hexane solutions for elemental analysis experiments.

$N^{N}$-(2-Chlorobenzylidene)-3-(2-phenyl-6-trifluoromethylpyrimidin-4-yl)propanoylhydrazide (11b)

Obtained $(72 \%)$ as a off-white solid, $\mathrm{mp} 148-150{ }^{\circ} \mathrm{C}$; ${ }^{1} \mathrm{H}$ NMR (400 MHz, $\left.\mathrm{CDCl}_{3}\right) \delta 3.37\left(\mathrm{~m}, 4 \mathrm{H}, 2 \mathrm{CH}_{2}\right), 7.24$ (s, 1H, H5), 7.30 (m, 2H, Ph), 7.42 (m, 5H, Ph), 7.95 (m, $1 \mathrm{H}, \mathrm{Ph}), 8.20$ (s, 1H, CH), 8.48 (m, 2H, Ph), 9.58 (s, 1H, $\mathrm{NH}) ;{ }^{13} \mathrm{C}$ NMR $\left(100 \mathrm{MHz}, \mathrm{CDCl}_{3}\right) \delta 30.2,32.3,113.9$ (q, $J$ $2.5 \mathrm{~Hz}), 120.8$ (q, J 276 Hz), 127.1, 128.5, 128.7, 129.3, 130, 131, 131.2, 131.4, 134.3, 136.5, 140.2, 155.8 (q, $J 36 \mathrm{~Hz}$ ), 165.1, 172.0, 174.3; HRMS (FTMS + pESI) $\mathrm{m} / \mathrm{z}$, calcd. for $\mathrm{C}_{21} \mathrm{H}_{16} \mathrm{ClF}_{3} \mathrm{~N}_{4} \mathrm{O}\left[\mathrm{MH}^{+}\right]: 433.1043$, found: $433.0967\left[\mathrm{MH}^{+}\right]$. Anal. calcd. for $\mathrm{C}_{21} \mathrm{H}_{16} \mathrm{ClF}_{3} \mathrm{~N}_{4} \mathrm{O} 432.70 \mathrm{~g} \mathrm{~mol}^{-1}$ : C, $58.27 ; \mathrm{H}$, 3.73 ; N, 12.94; found: $\mathrm{C}, 58.3 ; \mathrm{H}, 3.75 ; \mathrm{N}, 12.95$.

$N^{\prime}$-(2-Chlorobenzylidene)-3-(2-methylthio-6-trifluoromethylpyrimidin-4-yl)propanoylhydrazide (11c)

Obtained $(65 \%)$ as a yellow solid, mp $134-136{ }^{\circ} \mathrm{C}$; ${ }^{1} \mathrm{H} \mathrm{NMR}\left(400 \mathrm{MHz}, \mathrm{CDCl}_{3}\right) \delta 2.54$ (s, 3H, SMe), 3.23 (m, $\left.2 \mathrm{H}, \mathrm{CH}_{2}\right), 3.30\left(\mathrm{~m}, 2 \mathrm{H}, \mathrm{CH}_{2}\right), 7.21(\mathrm{~s}, 1 \mathrm{H}, \mathrm{H} 5), 7.30(\mathrm{~m}, 2 \mathrm{H}$, $\mathrm{Ph}), 7.37$ (m, 1H, Ph), $7.94(\mathrm{~m}, 1 \mathrm{H}, \mathrm{Ph}), 8.23(\mathrm{~s}, 1 \mathrm{H}, \mathrm{CH})$, $10,01$ (s, $1 \mathrm{H}, \mathrm{NH}) ;{ }^{13} \mathrm{C}$ NMR (100 MHz, $\left.\mathrm{CDCl}_{3}\right) \delta 14.1$, $30.2,31.9,111.2(\mathrm{q}, J 2.5 \mathrm{~Hz}), 120.4(\mathrm{q}, J 276 \mathrm{~Hz}), 127.1$, 130, 131, 131.1, 134.2, 134.7, 140.7, 155.3 (q, J $36 \mathrm{~Hz}$ ), 172.1, 173.9, 174.4; HRMS (FTMS + pESI) $\mathrm{m} / z$, calcd. for $\mathrm{C}_{16} \mathrm{H}_{14} \mathrm{ClF}_{3} \mathrm{~N}_{4} \mathrm{OS}\left[\mathrm{MH}^{+}\right]$: 403.0607 , found: 403.0608 $\left[\mathrm{MH}^{+}\right]$. Anal. calcd. for $\mathrm{C}_{16} \mathrm{H}_{14} \mathrm{ClF}_{3} \mathrm{~N}_{4} \mathrm{OS} 402.05 \mathrm{~g} \mathrm{~mol}^{-1}$ : C, $58.27 ; \mathrm{H}, 3.73 ; \mathrm{N}, 12.94$; found: C, 58.3; H, 3.75; N, 12.95 .

$N$-(2-Chlorobenzylidene)-3-(2-methyl-7-trifluoromethylpyrazolo[1,5-a]pyrimidin-5-yl)propa noylhydrazide (11d)

Obtained (73\%) as a white solid, mp $187-188{ }^{\circ} \mathrm{C}$; ${ }^{1} \mathrm{H}$ NMR (400 MHz, DMSO- $d_{6}$ ) $\delta 2.45$ (s, 3H, Me), 3.24 $\left(\mathrm{m}, 4 \mathrm{H}, 2 \mathrm{CH}_{2}\right), 6.64(\mathrm{~s}, 1 \mathrm{H}, \mathrm{H} 3), 7.40(\mathrm{~m}, 2 \mathrm{H}, \mathrm{Ph}), 7.50(\mathrm{~m}$, 2H, Ph), 7.93 (s, 1H, H6), 8.40 (s, 1H, CH), 11.52 (s, 1H, $\mathrm{NH}) ;{ }^{13} \mathrm{C}$ NMR (100 MHz, DMSO- $\left.d_{6}\right) \delta 13.2,29.8,31.8$,
96.2, 106.9 (q, $J 2.4 \mathrm{~Hz}), 119.5(\mathrm{q}, J 275.5 \mathrm{~Hz}), 131.4(\mathrm{q}$, $J 35.5 \mathrm{~Hz}), 126.6,127.5,129.8,131,131.4,132.8,138.8$, 149.3, 155.2, 161.2, 173.4; HRMS (FTMS + pESI) $\mathrm{m} / \mathrm{z}$, calcd. for $\mathrm{C}_{18} \mathrm{H}_{15} \mathrm{ClF}_{3} \mathrm{~N}_{5} \mathrm{O}\left[\mathrm{MH}^{+}\right]$: 410.0995 , found: 410.0995 $\left[\mathrm{MH}^{+}\right]$. Anal. calcd. for $\mathrm{C}_{18} \mathrm{H}_{15} \mathrm{ClF}_{3} \mathrm{~N}_{5} \mathrm{O} 409.79 \mathrm{~g} \mathrm{~mol}^{-1}$ : C, 47.71; H, 3.50; N, 13.91; found: C, 47.80; H, 3.55; N, 13.95 .

Ethyl 3-(6-trihalomethylpyrimidin-4-yl)propanoates from $\mathrm{N}$-acylhydrazones and TCCA

\section{General procedure}

$\operatorname{TCCA}(0.18 \mathrm{~g}, 0.75 \mathrm{mmol})$ at $25^{\circ} \mathrm{C}$ was added to a stirred solution of $N$-acylhydrazone 11 ( $3 \mathrm{mmol})$ in EtOH $(6 \mathrm{~mL})$. The solution was stirred for $30 \mathrm{~min}$; then the precipitate was filtered off $(\mathbf{1 2 b}, \mathbf{d})$ under vacuum. When the product did not precipitate, $\mathrm{EtOH}$ was evaporated and the residue was dissolved in $\mathrm{CH}_{2} \mathrm{Cl}_{2}(10 \mathrm{~mL})$, washed with water $(2 \times 10 \mathrm{~mL})$, and dried with $\mathrm{Na}_{2} \mathrm{SO}_{4}$. The solvent was evaporated, and product 12c was obtained as oil. The crystalline compounds were purified by recrystallization from hexane.

Ethyl 3-(2-phenyl-6-trifluoromethylpyrimidin-4-yl)propanoate (12b)

This compound was obtained (62\%) as yellow needles (hexane), mp 58-60 ${ }^{\circ} \mathrm{C}$; ${ }^{1} \mathrm{H}$ NMR (400.13 MHz, $\left.\mathrm{CDCl}_{3}\right) \delta 2.94\left(\mathrm{~m}, 2 \mathrm{H}, \mathrm{CH}_{2}\right), 3.24\left(\mathrm{~m}, 2 \mathrm{H}, \mathrm{CH}_{2}\right), 3.69$ (s, 3H, OMe), 7.36 (s, 1H, 5-H), 7.45-8.50 (m, 5H, Ph); ${ }^{13} \mathrm{C}$ NMR (100.62 MHz, $\left.\mathrm{CDCl}_{3}\right) \delta 31.1,32.3,51.7,113.7$ $\left(\mathrm{q},{ }^{3} J_{\mathrm{CF}} 2.7 \mathrm{~Hz}\right), 120.7\left(\mathrm{q}, J_{\mathrm{CF}} 275 \mathrm{~Hz}\right), 128.5,131.4$, 136.6, 155.6 (q, $\left.J_{\mathrm{CF}} 35.7 \mathrm{~Hz}\right), 164.9,171.2$, 173; HRMS (FTMS + pESI) $m / z$, calcd. for $\mathrm{C}_{16} \mathrm{H}_{15} \mathrm{~F}_{3} \mathrm{~N}_{2} \mathrm{O}_{2}\left[\mathrm{MH}^{+}\right]$: 325.1164, found $325.3053\left[\mathrm{MH}^{+}\right]$. Anal. calcd. for $\mathrm{C}_{16} \mathrm{H}_{15} \mathrm{~F}_{3} \mathrm{~N}_{2} \mathrm{O}_{2} 324.29 \mathrm{~g} \mathrm{~mol}^{-1}$ : C, 59.26; H, 4.66; N, 8.64; found: $\mathrm{C}, 59.3 ; \mathrm{H}, 4.65 ; \mathrm{N}, 8.7$.

Ethyl 3-(2-thiomethyl-6-trifluoromethylpyrimidin-4-yl) propanoate (12c)

This compound was obtained (52\%) as a brownish oil; ${ }^{1} \mathrm{H} \mathrm{NMR}\left(400.13 \mathrm{MHz}, \mathrm{CDCl}_{3}\right) \delta 1.21$ (t, 3H, Me), 2.57 (s, $3 \mathrm{H}, \mathrm{SMe}), 2.86\left(\mathrm{t}, 2 \mathrm{H}, \mathrm{CH}_{2}\right), 3.13\left(\mathrm{t}, 2 \mathrm{H}, \mathrm{CH}_{2}\right), 4.12(\mathrm{q}, 2 \mathrm{H}$, $\left.\mathrm{OCH}_{2}\right), 7.16$ (s, $\left.1 \mathrm{H}, \mathrm{H} 5\right) ;{ }^{13} \mathrm{C}$ NMR $\left(100.62 \mathrm{MHz}, \mathrm{CDCl}_{3}\right)$ $\delta 14.0,14.2,31.0,32.2,61.8,111.0\left(\mathrm{q},{ }^{3} J_{\mathrm{CF}} 2.7 \mathrm{~Hz}\right), 120.3$ $\left(\mathrm{q}, J_{\mathrm{CF}} 275 \mathrm{~Hz}\right), 155.3$ (q, $\left.J_{\mathrm{CF}} 36 \mathrm{~Hz}\right), 171.3,172.7,173.9$; HRMS (FTMS + pESI) $\mathrm{m} / z$, calcd. for $\mathrm{C}_{11} \mathrm{H}_{13} \mathrm{~F}_{3} \mathrm{~N}_{2} \mathrm{O}_{2} \mathrm{~S}$ [ $\left.\mathrm{MH}^{+}\right]$: 295.0728, found: $295.0727\left[\mathrm{MH}^{+}\right]$. Anal. calcd. for $\mathrm{C}_{11} \mathrm{H}_{13} \mathrm{~F}_{3} \mathrm{~N}_{2} \mathrm{O}_{2} \mathrm{~S} 294.29 \mathrm{~g} \mathrm{~mol}^{-1}$ : C, 44.89; H, 4.45; N, 9.52; found: C, 44.9; H, 4.46; N, 9.50 .

Ethyl 3-(2-methyl-7-trifluoromethylpyrazolo[1,5-a]pyrimidin5-yl)propanoate (12d)

Obtained as a yellowish solid (59\%), mp 120-123 ${ }^{\circ} \mathrm{C}$; 
${ }^{1} \mathrm{H}$ NMR $\left(400 \mathrm{MHz}, \mathrm{CDCl}_{3}\right) \delta 1.16(\mathrm{t}, 3 \mathrm{H}, J 6.4 \mathrm{~Hz}, \mathrm{Me})$, $2.46\left(\mathrm{~s}, 3 \mathrm{H}, \mathrm{CH}_{3}\right), 2.80\left(\mathrm{t}, 2 \mathrm{H}, J 7.0 \mathrm{~Hz}, \mathrm{CH}_{2}\right), 3.10(\mathrm{t}, 2 \mathrm{H}$, $\left.J 7.0 \mathrm{~Hz}, \mathrm{CH}_{2}\right), 4.06\left(\mathrm{q}, J 6.4 \mathrm{~Hz}, 2 \mathrm{H}, \mathrm{OCH}_{2}\right), 6.41(\mathrm{~s}, 1 \mathrm{H}$, $\mathrm{H} 3$ ), 6.90 (s, $1 \mathrm{H}, \mathrm{H} 5) ;{ }^{13} \mathrm{C}$ NMR (400 MHz, $\mathrm{CDCl}_{3}$ ) $\delta 14.1$, 14.6, 31.2, 32.5, 60.6, 96.8, 105.6 (q, $J 105.7 \mathrm{~Hz}), 119.5$ $(\mathrm{q}, J 275 \mathrm{~Hz}), 133.4(\mathrm{q}, J 37 \mathrm{~Hz}), 150.1,156.4,159.2$, 172.3; HRMS (FTMS + pESI) $\mathrm{m} / z$, calcd. for $\mathrm{C}_{13} \mathrm{H}_{15} \mathrm{~F}_{3} \mathrm{~N}_{3} \mathrm{O}_{2}$ $\left[\mathrm{MH}^{+}\right]$: 302.1116, found: $302.2018\left[\mathrm{MH}^{+}\right]$. Anal. calcd. for $\mathrm{C}_{13} \mathrm{H}_{14} \mathrm{~F}_{3} \mathrm{~N}_{3} \mathrm{O}_{2} 301.26 \mathrm{~g} \mathrm{~mol}^{-1}: \mathrm{C}, 51.83 ; \mathrm{H}, 4.68 ; \mathrm{N}, 13.95$; found: $\mathrm{C}, 51.9 ; \mathrm{H}, 4.70 ; \mathrm{N}, 14.0$.

\section{Supplementary Information}

Spectroscopic ${ }^{1} \mathrm{H}$ and ${ }^{13} \mathrm{CNMR}$ data of title compounds are provided in the supplementary information, available free of charge at http://jbcs.sbq.org.br as a PDF file.

\section{Acknowledgments}

The authors are grateful for the financial support from Conselho Nacional de Desenvolvimento Científico e Tecnológico (CNPq, Universal grant 6577818477962764-01), and Fundação de Amparo à Pesquisa do Estado do Rio Grande do Sul (FAPERGS, PqG grant 1016236). Fellowships from CNPq (J. L. Malavolta, D. L. de Mello) and CAPES (D. C. Flores) are also acknowledged.

\section{References}

1. Galletti, A. M. R.; Antonetti, C.; De Luise, V.; Licursi, D.; Di Nasso, N. N.; BioResources 2012, 7(2), 1824.

2. Pileidis, F. D.; Titirici, M.-M.; ChemSusChem 2016, 9, 562.

3. Timokhin, B. V.; Baransky, V. A.; Eliseeva, G. D.; Russ. Chem. Rev. 1999, 68, 73.

4. Meyer, F.; Chem. Commun. 2016, 52, 3077.

5. Kawai, H.; Shibata, N.; Chem. Rec. 2014, 14, 1024.

6. Oliveira, C. S.; Lira, B. F.; Barbosa-Filho, J. M.; Lorenzo, J. G. F.; Athayde-Filho, P. F.; Molecules 2012, 17, 10192.

7. El-Din Mohamed, F. S.; Hashem, A. I.; Swellem, R. H.; Nawwar, G. A. M.; Lett. Drug Des. Discovery 2014, 11, 304.

8. Patel, K. D.; Prajapati, S. M.; Panchal, S. N.; Patel, H. D.; Synth. Commun. 2014, 44, 1859.

9. Li, Y.; Zhu, H.; Chen, K.; Liu, R.; Khallaf, A.; Zhang, X.; Ni, J.; Org. Biomol. Chem. 2013, 11, 3979.

10. Liu, Q.; Chen, K.; Ni, J.; Li, Y.; Zhu, H.; Ding, Y.; RSC Adv. 2014, 4, 55445.

11. Abu-Zaied, M. A.; El-Telbani, E. M.; Elgemeie, G. H.; Nawwar, G. A. M.; Eur. J. Med. Chem. 2011, 46, 229.

12. Ningaiah, S.; Bhadraiah, U. K.; Doddaramappa, S. D.; Keshavamurthy, S.; Javarasetty, C.; Bioorg. Med. Chem. Lett. 2014, 24, 245.
13. Lee, S. H.; Seo, H. J.; Kim, M. J.; Kang, S. Y.; Lee, S.-H; Ahn, K.; Lee, M.-W.; Han, H.-K.; Kim, J.; Lee, J.; Bioorg. Med. Chem. Lett. 2009, 19, 6632.

14. Flores, A. F. C.; Flores, D. C.; Oliveira, G.; Pizzuti, L.; Silva, R. M. S.; Martins, M. A. P.; Bonacorso, H. G.; J. Braz. Chem. Soc. 2008, 19, 184.

15. Franco, M. S. F.; Casagrande, G. A.; Raminelli, C.; Moura, S.; Rossato, M.; Quina, F. H.; Pereira, C. M. P.; Flores, A. F. C.; Pizzuti, L.; Synth. Commun. 2015, 45, 692.

16. Flores, A. F. C.; Piovesan, L. A.; Pizzuti, L.; Flores, D. C.; Malavolta, J. L.; Martins, M. A. P.; J. Heterocycl. Chem. 2014, $51,733$.

17. Flores, A. F. C.; Malavolta, J. L.; Frigo, L. M.; Doneda, M.; Flores, D. C.; Synth. Commun. 2015, 45, 1198.

18. Malavolta, J. L.; Souto, A. A.; Mello, D. L.; Flores, D. C.; Flores, A. F. C.; J. Fluorine Chem. 2014, 158, 16.

19. Bonacorso, H. G.; Martins, M. A. P.; Bittencourt, S. R. T.; Lourega, R. V.; Zanatta, N.; Flores, A. F. C.; J. Fluorine Chem. 1999, 99, 177.

20. Flores, A. F. C.; Pizzuti, L.; Brondani, S.; Rossato, M.; Zanatta, N.; Martins, M. A. P.; J. Braz. Chem. Soc. 2007, 18, 1316.

21. Flores, A. F. C.; Rosales, P. F.; Malavolta, J. L.; Flores, D. C.; J. Braz. Chem. Soc. 2014, 25, 1439.

22. Majumdar, P.; Pati, A.; Patra, M.; Behera, R. K.; Behera, A. K.; Chem. Rev. 2014, 114, 2942.

23. Kudelko, A.; Jasiak, K.; Synthesis 2013, 45, 1950.

24. Henryk, F.; Anna, C.-J.; Waleria, R.; Henryk, T.; Phosphorus Sulfur Silicon Relat. Elem. 2000, 164, 67.

25. Zareef, M.; Iqbal, R.; Al-Masoudi, N. A.; Zaidi, J. H.; Arfan, M.; Shahzad, S. A.; Phosphorus Sulfur Silicon Relat. Elem. 2007, 182, 281.

26. Lo Monte, F.; Kramer, T.; Brodrecht, M.; Gu, J.; Pilakowski, J.; Fuertes, A.; Dominguez, J. M.; Plotkin, B.; Eldar-Fikelman, H.; Schmidt, B.; Eur. J. Med. Chem. 2013, 61, 26.

27. Amir, M.; Saifullah, K.; Akter, W.; Indian J. Chem., B: Org. Chem. Incl. Med. Chem. 2011, 50, 1107.

28. Murti, Y.; Mehrotra, V.; Pathak, D.; Int. J. Drug Discovery 2011, 2,659 .

29. Pore, D. M.; Mahadik, S. M.; Desai, U. V.; Synth. Commun. 2008, 38, 3121.

30. Mickevicius, V.; Vaickeliooniene, R.; Sapijanskaite, B.; Chem. Heterocycl. Compd. 2009, 45, 215. The procedure described was applied to $N^{\prime}$-acetyl-3-(2-phenyl-6-trifluoromethylpyrimidin4-yl)propanehydrazide (13b) obtained from acetylation of $\mathbf{3 b}$.

Submitted: December 20, 2016 Published online: March 24, 2017

FAPERGS/CAPES has sponsored the publication of this article. 